\title{
The onset of X-ray emission in young stellar objects
}

\section{A Chandra observation of the Serpens star-forming region ${ }^{\star}$}

\author{
G. Giardino ${ }^{1}$, F. Favata ${ }^{1}$, G. Micela ${ }^{2}$, S. Sciortino ${ }^{2}$, and E. Winston ${ }^{3}$ \\ 1 Astrophysics Division - Research and Science Support Department of ESA, ESTEC, Postbus 299, 2200 AG Noordwijk, \\ The Netherlands \\ 2 INAF - Osservatorio Astronomico di Palermo, Piazza del Parlamento 1, 90134 Palermo, Italy \\ 3 Harvard-Smithsonian Center for Astrophysics, 60 Garden Street, Cambridge, MA 02138, USA
}

Received 20 September 2006 / Accepted 2 November 2006

\section{ABSTRACT}

\begin{abstract}
Aims. To study the properties of X-ray emission from young stellar objects (YSOs) through their evolution from Class I to Class III and determine whether Class 0 protostars emit X-rays.

Methods. A deep Chandra X-ray observation of the Serpens star-forming region was obtained. The Serpens Cloud Core is ideally suited for this type of investigation, being populated by a dense and extremely young cluster whose members are found in all evolutionary stages, including six well-studied Class 0 sources.

Results. None of the six Class 0 protostars is detected in our observations, excluding the presence of sources with typical X-ray luminosities $z 0.4 \times 10^{30} \mathrm{erg} \mathrm{s}^{-1}$ (for column densities of the order of $4 \times 10^{23} \mathrm{~cm}^{-2}$, or $A_{\mathrm{V}} \sim 200$ ). A total of $85 \mathrm{X}$-ray sources are detected and the light curves and spectra of 35 YSOs are derived. There is a clear trend of decreasing absorbing column densities as one moves from Class I to Class III sources, and some evidence of decreasing plasma temperatures, too. We observe a strong, long-duration, flare from a Class II low-mass star, for which we derive a flaring loop length of the order of 20 stellar radii. We interpret the flaring event as originating from a magnetic flux tube connecting the star to its circumstellar disk. The presence of such a disk is supported by the detection, in the spectrum of this star, of $6.4 \mathrm{keV} \mathrm{Fe} \mathrm{fluorescent} \mathrm{emission.}$
\end{abstract}

Key words. ISM: clouds - ISM: individual objects: Serpens cloud - stars: pre-main sequence - X-rays: stars

\section{Introduction}

Young stellar objects (YSOs) are well-known X-ray sources, and $\mathrm{X}$-ray observations have become a routine tool for the study of star-forming regions. Beside its intrinsic astrophysics interest, $\mathrm{X}$-ray emission in the early stages of stellar evolution may have a significant influence on the circumstellar environment. As a ionizing source for the circumstellar accretion disk, X-ray emission may play an important role in regulating the coupling between the disk and the magnetic field and affect the chemistry of the disk itself. X-rays are an important component in the complex feedback processes regulating star-formation: Natta et al. (2006) speculate on the possibility that the observed spread in accretion rates observed in classical T Tauri stars (CCTS) of any given age (which is too large to be explained by "classical" accretion disk theory) might be explained by the large spread in X-ray luminosity among otherwise similar pre-main sequence stars, if the main source of disk ionization is indeed provided by the stellar X-ray emission. Additionally, as the hot X-ray emitting plasma needs to be confined by strong magnetic fields $(B \geq 100 \mathrm{G})$, tracing the $\mathrm{X}$-ray emission and its spatial extent provides a mean of locating strong magnetic fields, which (in the magnetospheric accretion model of low-mass YSOs) have a key role in channeling the accreting plasma and thus in regulating the accretion.

One open question regards the onset of X-ray emission: at which evolutionary phase do YSOs start to emit X-rays? Current

* Section 6, Tables 5 and 6, and Fig. 13 are only available in electronic form at http: //www . aanda.org evidence shows that X-ray emission is common (although perhaps not universal) in Class I sources (e.g Ozawa et al. 2005) Class I sources are protostars which already have an accretion disk but are still supplied with a relatively massive circumstellar envelope. In Class II sources (or CTTS, i.e. young stars actively accreting from a circumstellar disk, but without a circumstellar envelope) X-ray emission is an universal feature, as shown, for instance, by the deep Chandra observations of the Orion Nebula Cluster (Flaccomio et al. 2003; Preibisch et al. 2005). In more evolved sources, that are no longer accreting (Class III, or weaklined T Tauri stars - WTTS), X-ray emission is also an universal occurrence, with characteristics and luminosity similar to active main-sequence stars (e.g. Flaccomio et al. 2003; Preibisch et al. 2005).

Class 0 sources are young protostars at the beginning of the main accretion phase. Observationally, they are characterized by strong, centrally-condensed dust continuum emission at submillimeter wavelengths, powerful jet-like outflows, and very little emission shortward of $10 \mu \mathrm{m}$. According to the defintion given in Andre et al. (2000), their submillimeter luminosity (measured longward of $350 \mu \mathrm{m}$ ), should be greater than $0.5 \%$ of their bolometric luminosity - thereby suggesting that the envelope mass exceeds the central stellar mass. The evidence for X-ray emission from Class 0 protostars is still fragmentary. Tsuboi et al. (2001) claimed the discovery of X-rays from two highly embedded sources in the OMC-2/3 clouds, within a few arcsecs from the Class 0 candidates MMS 2 and MMS 3. The two detected sources show some Class 0 characteristics: a very large 
absorption $\left(N(\mathrm{H})=1-3 \times 10^{23} \mathrm{~cm}^{-2}\right)$, no near-IR counterparts, and associations with millimeter radio clumps. However, followup radio and near-IR observations by Tsujimoto et al. (2004) did not unambiguously classify either source as Class 0 , and associated one of them with emission from a proto-stellar jet originating in a Class I source.

Another claim for X-ray emission from Class 0 sources was made by Hamaguchi et al. (2005), who detected X-ray emission from 2 embedded sources in the R Coronae Australis starforming core. The two sources are associated with VLA centimeter radio sources and their X-ray spectra imply absorbing column densities of the order of $3 \times 10^{23} \mathrm{~cm}^{-2}$, or $A_{\mathrm{V}} \simeq$ $180 \mathrm{mag}$. One of the two sources, IRS 7W, does not appear to be a Class 0 source: according to Forbrich et al. (2006) the emerging picture is that of IRS $7 \mathrm{~W}$ being an infrared-detected, deeply-embedded protostar, probably a Class I or II source. The other source, IRS 7E, is proposed as a Class 0 source (Forbrich et al. 2006). The X-ray emission from this source displays strong variability (its luminosity varying between $0.2 \times 10^{31}$ and $2 \times 10^{31} \mathrm{erg} \mathrm{s}^{-1}$ on timescales of 3 to 30 months) and it is characterized by a high plasma temperature, $k T \simeq 3-4 \mathrm{keV}$.

Finally, Getman et al. (2006) have recently reported X-ray emission associated with the luminous Class 0/I protostar IRAS $21391+5802$ (in IC $1396 N$ ). Their Chandra data reveal a faint, extremely hard, X-ray source within $0.5^{\prime \prime}$ of BIMA 2, the millimeter counterpart of the IRAS source. From the 8 extracted photons they derive a median energy of the source of $6.0 \mathrm{keV}$ and infer an absorbing column density of $N(\mathrm{H}) \sim 10^{24} \mathrm{~cm}^{-2}$ $\left(A_{\mathrm{V}} \sim 500\right)$. The inferred intrinsic hard band luminosity corrected for absorption is $L_{\mathrm{X}} \sim 2 \times 10^{31} \mathrm{erg} \mathrm{s}^{-1}$, a high luminosity consistent with IRAS $21391+5802$ being an intermediate mass protostar with circumstellar mass $\sim 5 M_{\odot}$. The analysis of the photon arrival time suggests that IRAS $21391+5802$ was seen during the decay of a magnetic reconnection flare (Getman et al. 2006).

Thus, while some evidence of Class 0 X-ray emission is gathering, this is still patchy; the Serpens cloud is an ideal environment to try to investigate $\mathrm{X}$-ray emission from protostars further since it contains a set of well-studied, well-identified Class 0 sources and is nearby.

Here we present a $90 \mathrm{ks}$ Chandra observation of the Serpens Cloud Core. The Serpens cloud has been previously observed in $\mathrm{X}$-rays both with the ROSAT HRI and with XMM-Newton. The ROSAT observation was relatively short $(\simeq 19 \mathrm{ks})$ and resulted in the detection of 7 sources (Preibisch 1998), while a total of $45 \mathrm{X}$-ray sources were detected in the shorter XMM-Newton observation $(\simeq 12 \mathrm{ks}$, Preibisch 2003$)$. This last observation was combined by Preibisch (2004) with two other subsequent XMMNewton observations for a total exposure time of $\sim 52 \mathrm{ks}$. The present Chandra observation is therefore the deepest X-ray observation conducted to date of the Serpens region, allowing us to detect fainter sources (for a total number of 85) and to study the spectrum and the temporal variability of a significant number of YSOs in the region.

\subsection{Characteristics of the Serpens cloud}

The Serpens Cloud Core (diameter $\sim 6$ arcmin) is one of the more active, nearby star-forming regions and has been the subject of many observational studies during the last 15 years. Its distance is estimated at 260 pc by Straizys et al. (1996), using photometry of the brightest stars in the region. In the IR, the Serpens Cloud Core is dominated by two conical regions of diffuse emission extending out of a large disk-like absorption feature along the northeast-southwest direction (centered on the young star SVS 2); to the south, a filamentary, eye-shaped structure of intense emission is centered around SVS 20. The region is populated by a deeply-embedded and extremely young cluster whose members are found in many different evolutionary stages. Near-IR surveys identified more than 150 sources embedded in the cloud core (Eiroa \& Casali 1992; Sogawa et al. 1997; Giovannetti et al. 1998; Kaas et al. 2004;). Many of these sources were classified as Class II YSOs, by means of mid-IR Infrared Space Observatory (ISO) observations (Kaas et al. 2004); these observations also revealed a significant number of flat-spectrum sources and Class I protostars. Sub-millimeter, millimeter, and far-IR observations show that the cloud core is also populated by one of the richest collection of Class 0 objects and pre-stellar condensations (Casali et al. 1993; Hurt \& Barsony 1996; Testi \& Sargent 1998; Eiroa et al. 2005). The age of the Class II population of the cluster is estimated at 2-3 Myr (Giovannetti et al. 1998; Kaas et al. 2004), however on-going star formation is evident from the presence of protostars and pre-stellar condensations as well as several molecular outflows (Bally \& Lada 1983; White et al. 1995; Herbst et al. 1997; Davis et al. 1999) and the age of the Class I population is believed to be less than a few $10^{5}$ yr (Giovannetti et al. 1998; Kaas et al. 2004). Serpens was also the target of a Spitzer observation, as described in Sect. 2.1. We made use of the Spitzer data to provide a classification of the $\mathrm{X}$-ray sources detected in the Chandra observation. In particular, we used a Spitzer color-color diagram to determine whether a given YSO is Class I, II or III.

\section{Observations and data analysis}

The core of the Serpens cloud was observed with Chandra starting at 21:43 UT on June 19, 2004. The observation lasted for $91.4 \mathrm{ks}$ (although the scheduled duration was $100 \mathrm{ks}$ ). The data quality as received from the CXC was satisfactory, and we did not reprocess the data set. The image of the X-ray observation is shown in Fig.1, together with an IR image derived from the Spitzer observation in IRAC band $1(3.6 \mu \mathrm{m})$.

We performed the source detection on the unfiltered event list, using the Wavelet Transform detection algorithm developed at Palermo Astronomical Observatory (PWDETECT, available at http://oapa.astropa.unipa.it/progetti_ricerca/ PWDetect). A total of $85 \mathrm{X}$-ray sources were detected, of which we expect at most 1 to be spurious, based on the source significance limit with which PWDETECT was run. The coordinates and ACIS-I count rate of the detected sources are listed in Table 5. Note that sources 49 and 51 are unresolved from source 48 , as this is characterized by an elongated shape in the NE direction. Source 48 is the X-ray counterpart of SVS 20, the deeply-embedded IR double source located inside the "eye" structure at the center of the Serpens nebula (see Sect. 4.3 for more details).

Table 6 provides cross-identification of sources as obtained from the SIMBAD database (search radius of $5 \operatorname{arcsec}$ ).

For sufficiently bright X-ray sources (with count rate $\geq$ $0.5 \mathrm{cts} / \mathrm{ks}$ ) individual light curves and spectra were extracted using source and background regions defined in DS9 and CIAO 3.3 threads, which were also used for the generation of the relative response matrices. For these sources, spectral fits were performed using XSPEC 11.3 (see Sect. 3).

Figure 2 shows the $K$ vs. $J-K$ color-magnitude diagram for the X-ray sources listed in Table 5 which have a 2MASS counterpart. In the diagram $2 \mathrm{Myr}$ isochrones are shown for 4 values of extinction $\left(A_{K}=0,1,2,3\right)$. Reddening vectors have been 


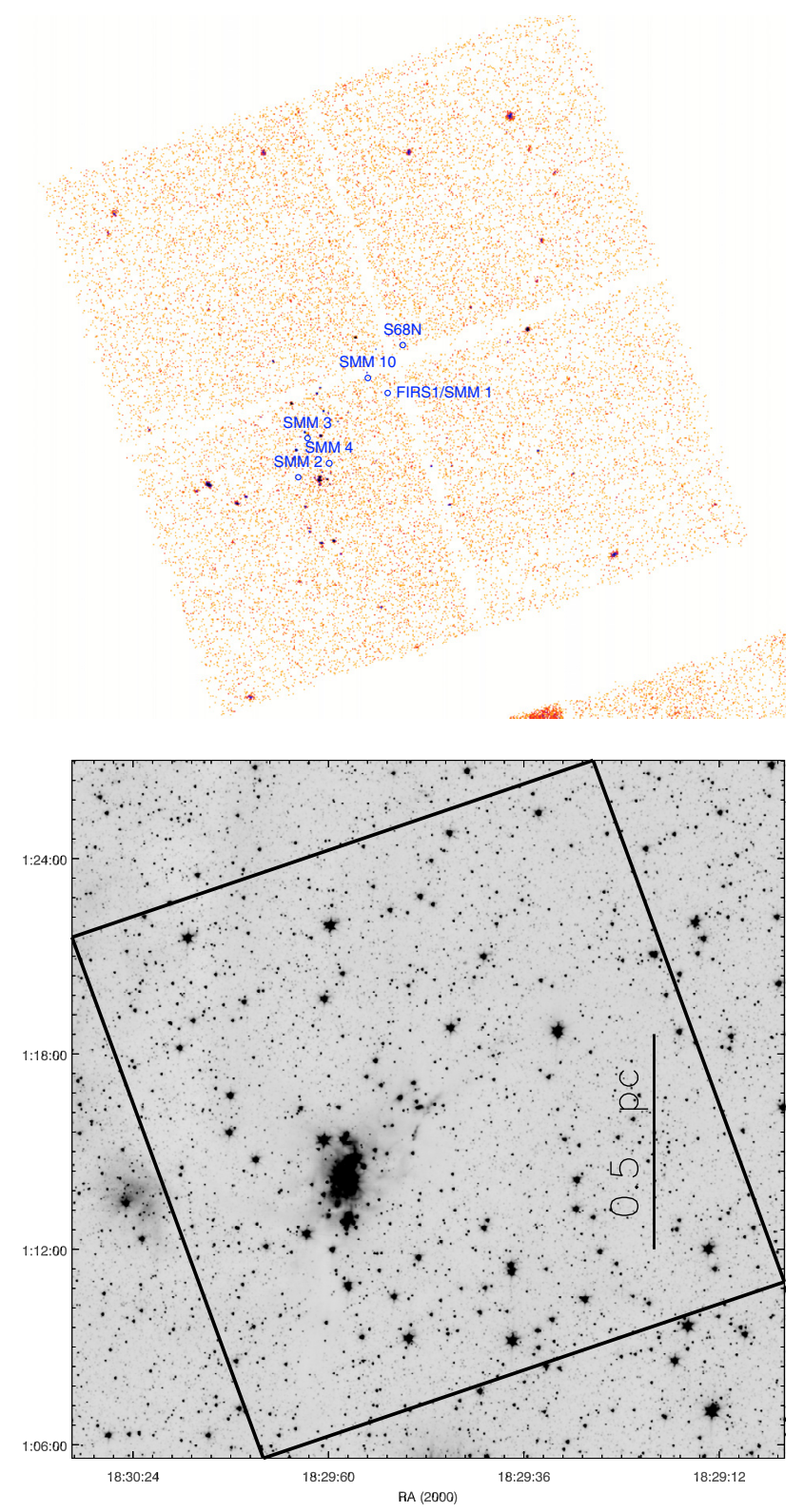

Fig. 1. Top - Chandra X-ray image of the Serpens Cloud Core; the positions of Class 0 sources are indicated. Bottom - Spitzer IRAC band 1 view of the Serpens (the outline gives the Chandra FOV).

computed following the relation $A_{K}=R_{K} \times E(J-K)$, where $E(J-K)$ is the colour excess $\left(E(J-K)=(J-K)-(J-K)_{0}\right)$ and $R_{K}=0.66$ (Rieke \& Lebofsky 1985). The isochrones are from Siess et al. (2000), for a metal abundance $Z=0.02$ plus overshooting, shifted to the distance of the Serpens $(260 \mathrm{pc})$. Although, various simplifying assumptions apply to the model isochrones (e.g. they include neither rotation nor accretion) and evolutionary models for pre-main sequence stars are not yet well established, they allow a mass estimate for most of our sources to be derived. The X-ray sample comprises a significant number of very low-mass stars $\left(M<0.1 M_{\odot}\right), 6$ intermediate mass stars and the highly reddened double source SVS 20 (source 48).

The $J-H$ versus $H-K$ color-color diagram in Fig. 3 allows normally reddened stars to be discriminated from stars with IR excess (indicative of warm circumstellar dust in addition to a reddened photosphere). The majority of stars with high

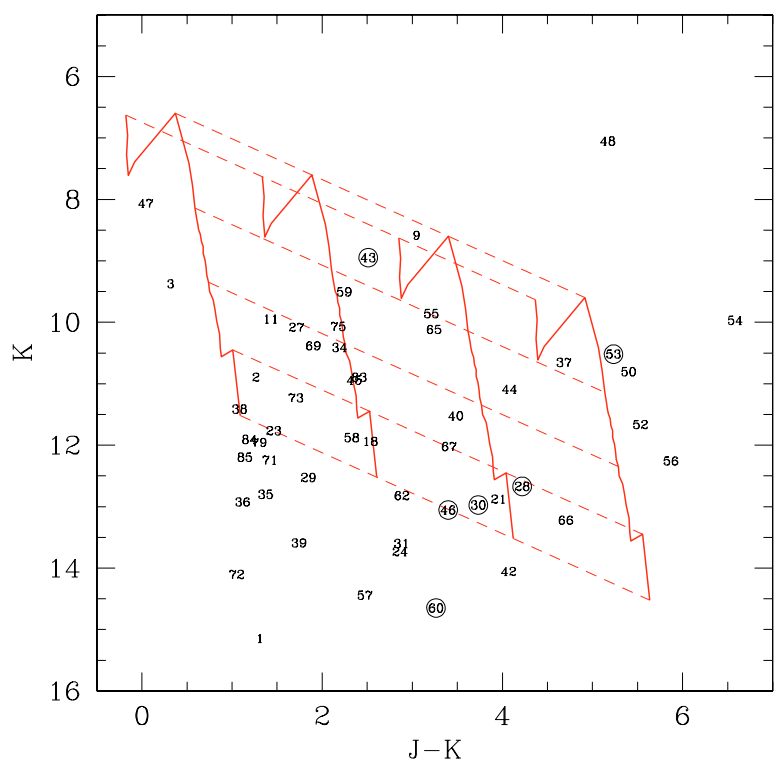

Fig. 2. Color magnitude diagram for the X-ray sources with 2MASS counterparts (Table 5). Theoretical $2 \mathrm{Myr}$ isochrones are also shown for different extinction coefficients (from left to right $A_{K}=0,1,2,3$ ). The dashed lines indicate the reddening vector for stars of constant mass (from top to bottom 3, 7, 2.2, 1, 0.2, and $0.1 M_{\odot}$ ). Circled numbers indicates Class I sources: their position in the diagram should not be compared to the 2 My isochrones, as their ages is estimated to be less than $\sim 10^{5} \mathrm{yr}$.

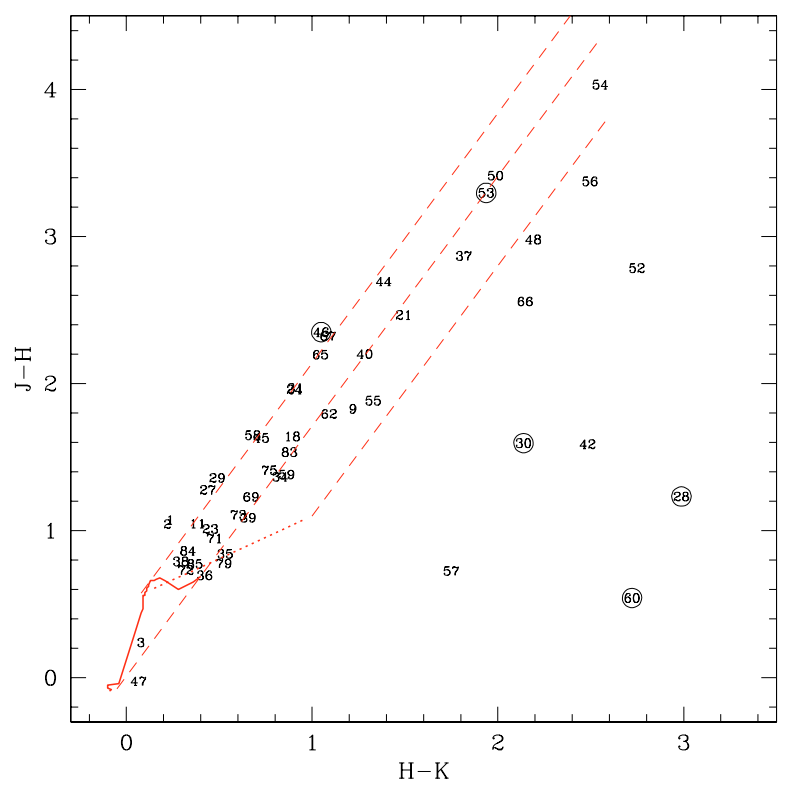

Fig. 3. Color-color diagram for the X-ray sources in Table 5 with 2MASS counterpart. The solid line at lower-left is the theoretical $2 \mathrm{Myr}$ isochrone. The dotted line yields the locus of dereddened colours of classical T Tauri stars according to Meyer et al. (1997). The dashed lines mark the reddening band for normally reddened stars. Class I sources are identified by a circle.

IR excess are indeed Class I sources or Flat spectrum sources (Class I/II).

\subsection{Spitzer data}

A detailed description of the processing and data reduction of the Spitzer infrared data can be found in Winston et al. (2006). 


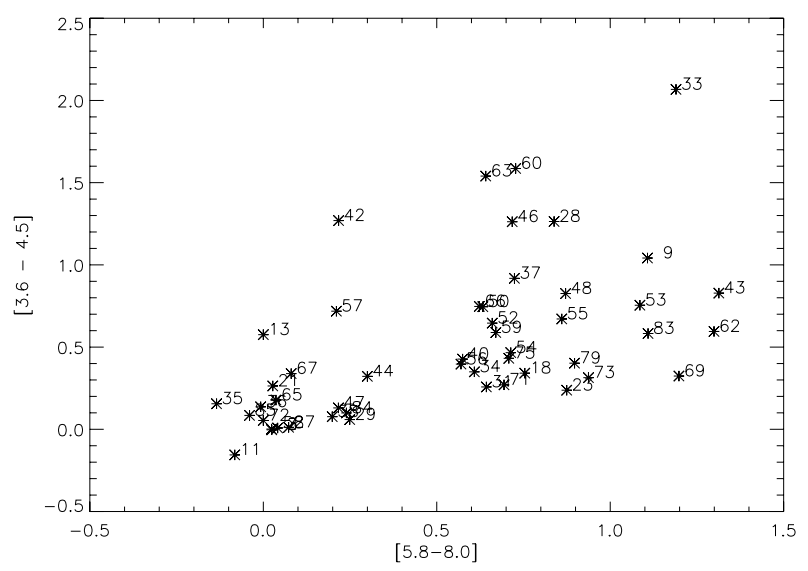

Fig. 4. Spitzer color-color diagram for the X-ray sources detected in all four Spitzer bands.

The Spitzer Space Telescope observed the Serpens region at infrared wavelengths from 3.6-70 $\mu \mathrm{m}$ as part of the Guaranteed Time Observations (GTO) program PID 6. The InfraRed Array Camera (IRAC; Fazio et al. 2004) observed the Serpens Cloud Core at 3.6, 4.5, 5.8, $8.0 \mu \mathrm{m}$, while the Multiband Imaging Photometer for Spitzer (MIPS; Rieke et al. 2004) provided observations at 24 and $70 \mu \mathrm{m}$. The IRAC bands detected sources below the hydrogen burning limit in Serpens. The IRAC and MIPS data were combined with near-IR $J, H$, and $K$ band data from 2MASS. The overlap region for these eight bands covered the entire Chandra field of view.

The classification of the sources was carried out using the slope of the Spectral Energy Distributions (SEDs) between the wavelengths $3.6 \mu \mathrm{m}$ and $8.0 \mu \mathrm{m}$, as a measure of the excess IR emission from circumstellar material. Sources were classified as follows: Class I are sources with a slope greater than 0. Flat spectrum (Class I/II) sources have "flat" slopes between 0.0 and -0.5 . Class II YSOs show excess emission from a circumstellar disk, with a slope between -0.5 and $\sim-3.5$. Class III sources exhibit photospheric emission, with slopes of $\sim-3.5$. Transition Disk sources (Class II/III) show photospheric emission shortwards of $8.0 \mu \mathrm{m}$, with excess emission at $24 \mu \mathrm{m}$. In this case we only observe disk emission from the outer regions of the disk, indicative of disks with large inner holes. These may be due to grain growth or disk clearing by (proto) planets. A more detailed description of the classification is given in Winston et al. (2006).

The X-ray and infrared lists were merged with a positional accuracy of $\sim 1$ arcsec. Of the $85 \mathrm{X}$-ray sources, 72 were detected in at least one of the IR bands. Of these 72, 56 could be classified by our infrared data. The remaining 16 sources were detected in too few Spitzer bands to construct their SEDs and were faint (>15 mag) at $3.6 \mu \mathrm{m}$. We consider these objects likely to be background galaxies. The 56 brighter objects were classified as follows: 9 Class I, 9 Flat Spectrum, 19 Class II, 2 Transition Disks, and 17 Class III sources.

\section{Results}

\subsection{The lack of $X$-ray emission from Class 0 sources}

The Serpens cloud has been the subject of many observational studies in the submm and far IR, which have identified a number of Class 0 sources. Casali et al. (1993) published the first list of submm sources without near-IR counterparts in the Serpens (FIRS1/SMM1, SMM2, SMM4, SMM3) and Hurt \& Barsony (1996) indicated these four objects plus S68N (SMM 9) to be
Table 1. Class 0 and Class $0 / \mathrm{I}$ sources in the field of view of our Chandra ACIS observation. Their ISO counterpart is also indicated. Coordinates are from: Hogerheijde et al. (1999) for FIRS1, SMM2, SMM3, and SMM4; Wolf-Chase et al. (1998) for S68N and Davis et al. (1999) for SMM10. Hogerheijde et al. (1999) indicated SMM2 as starless.

\begin{tabular}{l|cccc}
\hline \hline Name & RA & Dec & $\begin{array}{c}\text { ISO } \\
\text { id }\end{array}$ & Cl. \\
\hline FIRS1/SMM1 & 182949.7 & +011521 & - & 0 \\
SMM2 & 183000.4 & +011250 & - & $0 ?$ \\
SMM3 & 182959.3 & +011400 & - & 0 \\
SMM4 & 182956.7 & +011315 & 308 & $0 / I$ \\
S68N/SMM9 & 182947.9 & +011647 & 241 & $0 / I$ \\
SMM10 & 182952.1 & +011548 & 270 & $0 / I$ \\
\hline
\end{tabular}

Class 0 . These objects were also detected in a later survey of $3 \mathrm{~mm}$ radio emission by Testi \& Sargent (1998), where a total of 32 sources in a $5.5 \times 5.5$-arcmin area, were identified. Wolf-Chase et al. (1998) also identified S68N as a Class 0 object and reported a more accurate position for it. Hogerheijde et al. (1999) confirmed the Class 0 status of FIRS1, SMM3 and SMM4 but suggested that SMM2 may not contain a central protostar. Davis et al. (1999) obtained wide-field submm continuum and CO $J=2-1$ observations of the Serpens Cloud Core. They detected all the above mentioned sources plus SMM8, SMM10 and SMM11, however for SMM8 and SMM11 they were unable to derive a spectral index ${ }^{1}$. Davis et al. (1999) also suggested that these Serpens submm source may be "warm", late Class 0 or early Class I objects.

Eiroa et al. (2005) searched for $3.5 \mathrm{~cm}$ VLA sources in Serpens and, together with eighteen more evolved sources, they detected FIRS1, SMM4, SMM9/S68N, and SMM10. They also searched for ISO counterparts and suggested that these four sources had an ISO counterpart; they noted that the Class 0 classification given in the literature, for these four sources, is based on (sub-)millimetric observations (Hurt \& Barsony 1996; WolfChase et al. 1998; Hogerheijde et al. 1999), while the Class I classification is taken from ISO mid-IR observations (Kaas et al. 2004). In the case of FIRS1, however, the cross-identification with ISO objects $258 \mathrm{a}$ and $258 \mathrm{~b}$ is uncertain, since ISO objects $258 \mathrm{a}$ and $258 \mathrm{~b}$ lie $\gtrsim 9$ arcsec away from the position of the $\mathrm{mm} / \mathrm{submm}$ source and, as discussed by Kaas et al. (2004), they could be scattered light from the far-IR source FIRS1. Kaas et al. (2004) also exclude ISOCAM detections for SMM2 and SMM3 within the positional uncertainties.

Table 1 provides the positions for this sample of Class 0 or $0 /$ I sources which fall in the ACIS field of view of our Serpens observation. None of these 6 sources was detected in the recent Spitzer observation confirming the high ratio of sub-millimiter to mid-IR luminosity for these sources, and thereby their Class 0 nature.

An important result from the present study is that none of the six Class 0 sources in Table 1 are individually detected as X-ray sources. This non-detection can be translated in an estimate of the absorbing column density necessary to absorb a source's emission to the level where it would become undetectable in our data (below $\sim 0.1 \mathrm{cts} / \mathrm{ks}$, as per Table 5), assuming a spectrum and luminosity for the source. We used as proxy the X-ray spectrum of a Class I source in our field, source 60 (with $0.7 \mathrm{cts} / \mathrm{ks}$ ), and increased the absorbing column density of its best-fit model

\footnotetext{
1 Due to the lack of 3-mm fluxes from Testi \& Sargent (1998) for these two sources.
} 


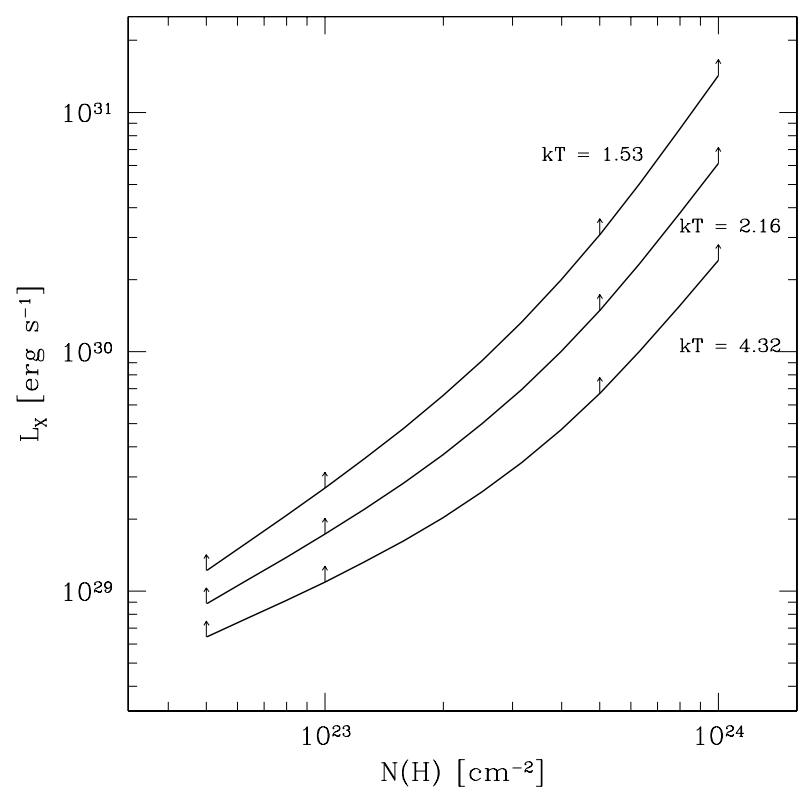

Fig. 5. Minimum X-ray luminosity required for a source to be detectable in our observations, as a function of the absorbing column density, for different temperatures of the emitting plasma. The curves were obtained using the PIMMS software at HEASARC, assuming for the emitting plasma a Raymond-Smith model with $Z=0.2 Z_{\odot}$.

to the value at which the model predicted source counts would be $\sim 0.1 \mathrm{ks}^{-1}$, i.e. our detection limit. Source 60 would become undetectable in our data behind an absorbing column density of $N(\mathrm{H}) \sim 40 \times 10^{22} \mathrm{~cm}^{-2}$ or $A_{\mathrm{V}} \sim 200$. Source 60 has a best fit $k T$ value of $2.4 \mathrm{keV}$ and an (intrinsic) luminosity of $10^{30} \mathrm{erg} \mathrm{s}^{-1}$, which are typical for Class I sources. In the sample of five Class I sources of $\rho$ Oph compiled by Ozawa et al. (2005), the average values of $k T$ and $L_{\mathrm{X}}$ are $3.2 \mathrm{keV}$ and $2.9 \times 10^{30} \mathrm{erg} \mathrm{s}^{-1}$. Therefore, in the assumption that Class 0 sources have X-ray characteristics similar to Class I objects, source 60 is a good proxy.

As already mentioned, Tsuboi et al. (2001) and Hamaguchi et al. (2005) derive column densities of the order of $(10-30) \times 10^{22} \mathrm{~cm}^{-2}$ in their X-ray observations of highly embedded YSOs. Since these objects appear to have higher plasma temperatures than source $60(k T \sim 3-4 \mathrm{keV})$ and similar luminosity, than we can exclude the possibility that X-ray sources similar to the one reported by the above authors are embedded within the $\mathrm{mm} / \mathrm{submm}$ sources of Table 1. As shown in Fig. 5, a source with a higher plasma temperature than source 60 and the same luminosity would have to be screened by an absorbing column density higher than $40 \times 10^{22} \mathrm{~cm}^{-2}$ for its count rate to be below our detection threshold. For comparison, a source with $k T=4.3 \mathrm{keV}$ and $L_{\mathrm{X}}=10^{30} \mathrm{erg} \mathrm{s}^{-1}$ would be undetectable in our data only if screened by an absorbing column density $\gtrsim 60 \times 10^{22} \mathrm{~cm}^{-2}\left(A_{\mathrm{V}} \gtrsim 300 \mathrm{mag}\right)$.

A source with characteristics similar to IRAS $21391+5802$, reported by Getman et al. (2006), would have also been well visible in our observation, its properties $\left(N(\mathrm{H}) \sim 10^{24}, k T=\right.$ $6.0 \mathrm{keV}$ and $L_{\mathrm{X}} \sim 2 \times 10^{31} \mathrm{erg} \mathrm{s}^{-1}$ ) implying in ACIS a count rate of $1.0 \mathrm{ks}^{-1}$. If the high luminosity and plasma temperature of IRAS $21391+5802$ are, however, the result of a flare and the more common situation is the one in which a lower luminosity, lower plasma temperature, source (e.g. with $L_{X}=10^{30} \mathrm{erg} \mathrm{s}^{-1}$ and $k T=2-4 \mathrm{keV}$ ) is hidden behind 500 mag of extinction, then our observation is not sensitive enough to detect such a source (see Fig. 5).

Of course, we cannot exclude the possibility that an X-ray emitting source with properties similar to the ones of source 60 is hiding beneath a column of absorbing material higher than $40 \times 10^{22} \mathrm{~cm}^{-22}$ or that these Class 0 sources are weaker sources. In order to derive a more sensitive upper limit for the typical Class 0 source in the Serpens Cloud Core, we registered and co-added the $\mathrm{X}$-ray photons from $200 \times 200$ pixels $(98 \times$ 98 arcsec) regions around each of the sources, centered on the coordinates given in Table 1 . Since there are six such regions, the result of this operation is equivalent (under the assumption that the six Class 0 sources are all similar to each other) to an observation of a single Class 0 source for an integration time six times longer than our original observation, i.e. $\sim 540 \mathrm{ks}$. The image of the "coadded" event list, resulting from this operation, is shown in Fig. 6 for the two energy intervals $\Delta E=0.5-8.0 \mathrm{keV}$ and $\Delta E=4.0-8.0 \mathrm{keV}^{3}$. No source appears to be present in the centre of the coadded image, within a $5^{\prime \prime}$ radius, corresponding to the positional uncertainties of the $\mathrm{mm} / \mathrm{submm}$ objects. The total number of counts within the $3^{\prime \prime}$-radius central area is 15 (a $3^{\prime \prime}$ radius being typical of source extraction regions for ACIS data), completely consistent with the background level for the coadded event list. The measured average background level in the coadded event file is 0.5 cts $\operatorname{arcsec}^{-2}$, i.e. $14 \pm 4$ counts in a circular area with a $3^{\prime \prime}$ radius. This agrees well with the level of background in the original image, $0.083 \mathrm{cts} \mathrm{arcsec}^{-2}$, indicating that the "coadded" image does not suffer from significant systematics.

In the coadded image, a source at $6 \sigma$ level $^{4}$ above the background would have implied 41 counts within a 3 "-radius area, corresponding to a source count rate of $0.04 \mathrm{cts} / \mathrm{ks}$. The fact that there is no such a source sets a more stringent upper limit on the typical level of $\mathrm{X}$-ray emission from these Class 0 sources of $L_{\mathrm{X}}<0.4 \times 10^{30} \mathrm{erg} \mathrm{s}^{-1}$ (in the above assumption of $N(\mathrm{H})=$ $40 \times 10^{22} \mathrm{~cm}^{-2}$ and $\left.k T=2.3 \mathrm{keV}\right)$.

The median luminosity of the YSOs for which spectra have been studied in the present work (32 out of 35 - as three were undergoing a strong flare) is $0.4 \times 10^{30} \mathrm{erg} \mathrm{s}^{-1}$, the same as the upper limit on the typical X-ray luminosity of the Class 0 sources, implying that if the Class 0 sources in the Serpens cloud are indeed X-ray sources, then they have luminosities below the level typical of the other YSO in the region (or they are hidden behind an absorbing column density higher than $40 \times 10^{22} \mathrm{~cm}^{-2}$ ).

\subsection{Spectral characteristics of the $X$-ray-bright YSOs}

A spectral and timing analysis was carried out on all sufficiently bright sources (count rate $>0.5 \mathrm{cts} / \mathrm{ks}$ ). The spectra and light curves of all the sources brighter than $2.0 \mathrm{cts} / \mathrm{ks}$ are shown in Figs. 10 and 13, the results of the spectral analysis for these source are summarised in Table 2. The results of the spectral analysis for some of the sources with count rate between 0.5 and $2.0 \mathrm{cts} / \mathrm{ks}$ are also summarised in Table 2 . For these weaker sources, only the results for the sources with Spitzer

\footnotetext{
2 In $\rho$ Oph, Motte et al. (1998) derives, for compact cores, molecular hydrogen column densities of $N(\mathrm{H} 2)=(10-80) \times 10^{22} \mathrm{~cm}^{-2}$, corresponding to $A_{\mathrm{V}}=100-800$.

3 If Class 0 or Class 0/I sources have high plasma temperatures, as in the case reported by Getman et al. (2006), then they could be more easily detectable in an hard-band image.

4 5-6 $\sigma$ above background being the significance level of the weaker sources in our list.
} 

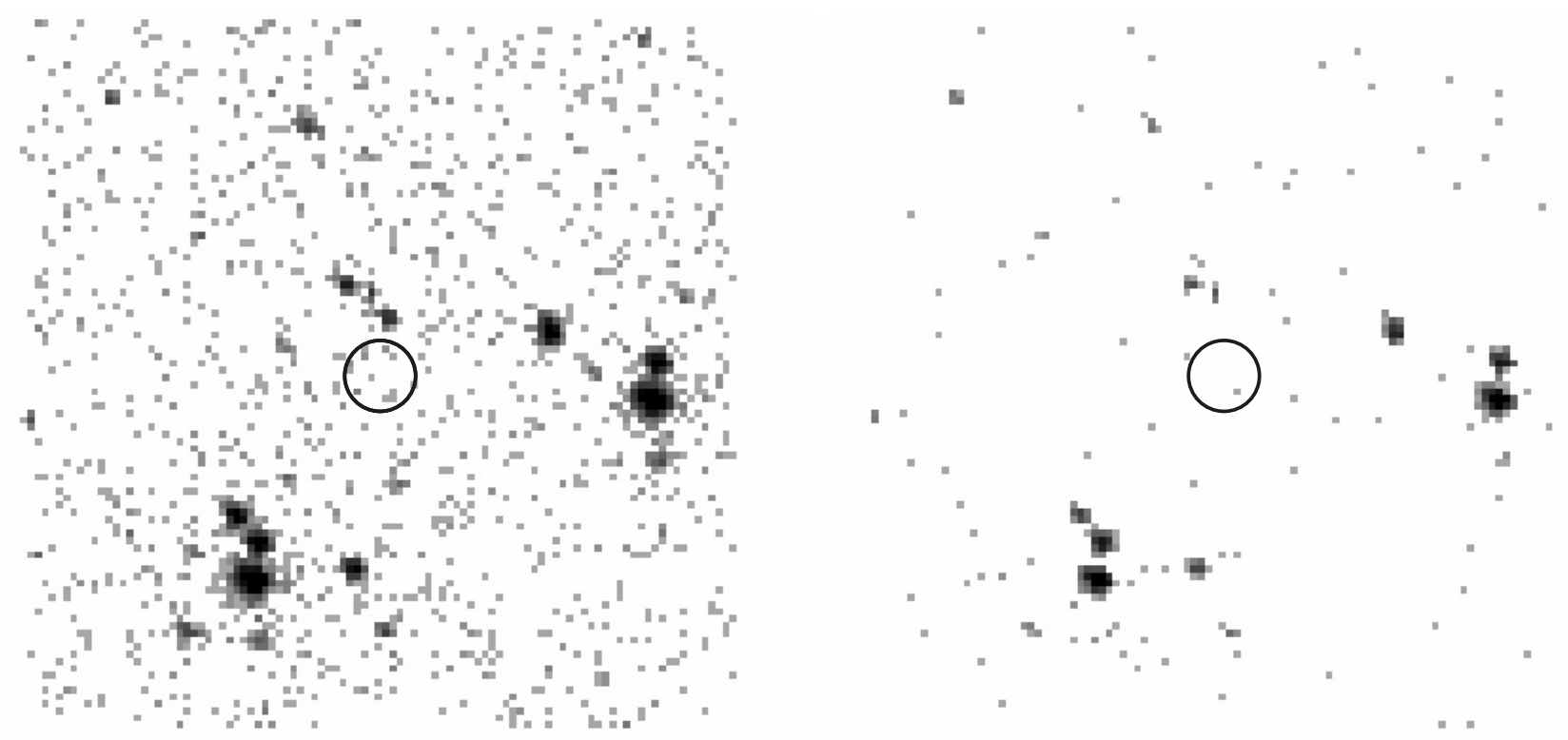

Fig. 6. The coadded image obtained by adding ACIS events from six regions of $200 \times 200$ pixels centered on the position of the Class 0 sources, for the energy intervals $\Delta E=0.5-8.0 \mathrm{keV}$ (left) and $\Delta E=4.0-8.0 \mathrm{keV}$ (right). The circular region in the center is $5^{\prime \prime}$, corresponding to positional uncertainties of the $\mathrm{mm} / \mathrm{submm}$ sources. No X-ray source is present within this area indicating that the Class 0 sources in our sample are unlikely to be X-ray sources with intensities just below our detection threshold. The sources present in the image are genuine sources falling by chance near the position of one of the Class 0 sources, which have been "coadded".

classification are reported. In the sample of sources stronger than $0.5 \mathrm{cts} / \mathrm{ks}$, there are no sources with a ISO counterpart but no Spitzer data, while there is a number of sources observed by Spitzer for which there are no ISO counterparts, so in Table 2 we used only the classification derived from the Spitzer observation. The sources are subdivided in the table according to their classification.

The foreground absorption toward the Serpens complex can be estimated using the galactic extinction law of Bahcall \& Soneira (1980),

$A_{\mathrm{V}}=\alpha_{\text {inf }}(1-\exp (-z / 100))$

where $\alpha_{\text {inf }}=0.15 / \sin b, z=10^{((D M+5) / 5)} \sin b, b$ being the galactic latitude and $D M$ the distance modulus. Using the distance to Serpens of $260 \mathrm{pc}(D M=7)$ and its latitude $(b=5.39)$ one derives a minimum value of $A_{\mathrm{V}}=0.3$ or $N(\mathrm{H})=0.06 \times 10^{22} \mathrm{~cm}^{-2}$. The source with the lowest value of absorbing column density is source 38 for which $N(\mathrm{H})=0.05 \pm 0.06 \times 10^{22} \mathrm{~cm}^{-2}$, consistent with all the YSOs for which spectra and light curves have been studied here being part of the Serpens complex (rather than being foreground objects).

Figure 7 shows the values of the absorbing column densities vs. the plasma temperatures of the sources in Table 2, except for the sources undergoing a strong flare $(66,79$ and 44) or unlikely to be stars $(6,13$ and 70), which have been omitted. A trend of decreasing absorbing column densities from Class I to Class III is clearly present. There is a lack of low-plasma-temperature high-absorption sources, which is expected, as with low plasma temperature higher luminosities are necessary in order to see a source through higher column densities. There is also, possibly, a lack of sources in the lower-right region of the diagram (where sources have a high plasma temperature and small absorption, and are thus easy to detect), hinting at an evolutionary effect from the Class I (high X-ray temperature, high extinction) to the Class III stage (lower X-ray temperature and extinction). Table 3 lists the median values of $N(\mathrm{H})$ and $k T$ for the different classes from which the trend of decreasing absorbing column density from Class I to Class III is clear and a trend of decreasing plasma temperature is also somewhat present (although less clear). Note also that, since these median values are based on detected sources only (we did not include upper limits, because of the lack of a parent catalogue), the trend in $k T$ could be due to a selection effect (i.e. Class I/II soft sources, being very absorbed, are not detected).

The diagram in Fig. 7 is similar to the one derived by Ozawa et al. (2005) for the $\rho$ Oph cloud core. They also note a lack of sources in the high-plasma-temperature small-absorption region of the diagram and interpret this as evidence of an evolutionary effect. A similar effect is noted by Flaccomio et al. (2006) in their study of the NGC 2264 star-forming complex.

As shown in Table 3, no evolutionary pattern is seen in the sources' luminosities. The higher X-ray luminosities of Class I YSOs likely being due to a selection effect, since only the brightest sources are detected behind higher column densities.

\subsection{Temporal variability of the the X-ray bright YSOs}

The majority of the YSOs with count rate $>0.5 \mathrm{cts} / \mathrm{ks}$, i.e. bright enough for their light curves to be analysed, shows significant variability, although with different characteristics. Three sources (44, 66 and 79) undergo a flare, with that of source 79 being the longest and most intense. This flare is analysed and discussed in detail in Sect. 4.7.

To evaluate the presence of X-ray variability, the Kolmogorov-Smirnov test, which measures the maximum deviation of the integral photon arrival times from a constant source model, was applied to all the brighter X-ray sources (count rate $>0.5 \mathrm{cts} / \mathrm{ks}$ ). The results, in terms of constancy probability, are given in Table 2 . The majority of sources, 21 out of 38 , has a probability of constancy below $2 \%$. Of these 21 , 19 are classified as YSOs (sources 13 and 70 are unlikely to be stars). 
Table 2. Best-fit spectral parameters for $38 \mathrm{X}$-ray sources with count rate greater than $0.5 \mathrm{cts} / \mathrm{ks}$. Results for all sources with count rate greater than $2 \mathrm{cts} / \mathrm{ks}$ are given; for the sources with rate between $0.5-2.0 \mathrm{cts} / \mathrm{ks}$, only results for sources which have a counterpart with a Spitzerderived YSO class are reported. $E M$ is the emission measure, $P$ the null-hypothesis probability of the fit, and $L_{\mathrm{X}}$ is the $\mathrm{X}$-ray luminosity derived from the "unabsorbed" X-ray flux. Units are $N_{22}=10^{22} \mathrm{~cm}^{-2}, E M_{53}=10^{53} \mathrm{~cm}^{-3}, F_{-13}=10^{-13} \mathrm{erg} \mathrm{cm}^{-2} \mathrm{~s}^{-1}$, and $L_{30}=10^{30} \mathrm{erg} \mathrm{s}^{-1}$. The spectral fits were carried out in the energy range $0.3-7.5 \mathrm{keV}$ unless otherwise indicated. $C_{\mathrm{KS}}$ gives the probability of constancy from the Kolmogorov-Smirnov test for the sources' light curves.

\begin{tabular}{|c|c|c|c|c|c|c|c|c|c|}
\hline $\mathrm{Src}$ & $N(\mathrm{H})$ & $k T$ & $Z$ & $E M$ & $\chi^{2}$ & $P$ & $F_{\mathrm{X}}$ & $L_{\mathrm{X}}$ & $C_{\mathrm{KS}}$ \\
\hline & $N_{22}$ & $\mathrm{keV}$ & $Z_{\odot}$ & $E M_{53}$ & & & $F_{-13}$ & $L_{30}$ & \\
\hline \multicolumn{10}{|l|}{ Class I } \\
\hline 32 & $15.38 \pm 10.47$ & $2.21 \pm 2.35$ & 0.30 (froz.) & $2.01 \pm 9.01$ & 1.13 & 0.34 & 0.27 & 2.20 & 0.03 \\
\hline 60 & $7.4 \pm 2.54$ & $2.36 \pm 1.32$ & 0.3 (froz.) & $0.93 \pm 1.67$ & 0.26 & 1 & 0.21 & 1.01 & 0.11 \\
\hline 61 & $4.00 \pm 0.94$ & $3.11 \pm 1.39$ & 0.30 (froz.) & $0.54 \pm 0.56$ & 1.23 & 0.20 & 0.24 & 0.68 & 0.05 \\
\hline \multicolumn{10}{|l|}{ Class I/II } \\
\hline $13^{*}$ & $4.34 \pm 0.81$ & $21.82 \pm 32.48$ & 0.3 (froz.) & $0.42 \pm 0.45$ & 0.8 & 0.91 & 1.07 & 1.7 & $0.1 \mathrm{e}-5$ \\
\hline $37^{\dagger}$ & $1.91 \pm 0.83$ & $2.26 \pm 1.3$ & 0.3 (froz.) & $0.14 \pm 0.19$ & 1.2 & 0.3 & 0.06 & 0.15 & $0.9 \mathrm{e}-4$ \\
\hline 48 & $3.6 \pm 0.32$ & $2.5 \pm 0.34$ & 0.3 (froz.) & $3.5 \pm 1.3$ & 0.84 & 0.91 & 1.26 & 3.9 & $0.1 \mathrm{e}-4$ \\
\hline 53 & $4.28 \pm 0.41$ & $3.83 \pm 0.78$ & 0.3 (froz.) & $2.65 \pm 1.03$ & 0.63 & 1 & 1.39 & 3.47 & 0 \\
\hline $55^{\dagger}$ & $1.05 \pm 0.3$ & $1.22 \pm 0.26$ & 0.3 (froz.) & $0.46 \pm 0.34$ & 0.47 & 0.98 & 0.14 & 0.44 & 0.45 \\
\hline $66^{\dagger} \mathrm{f}$ & $1.75 \pm 0.75$ & $4.33 \pm 4.25$ & 0.3 (froz.) & $0.16 \pm 0.15$ & 1.01 & 0.44 & 0.13 & 0.22 & 0 \\
\hline $83^{\dagger}$ & $0.81 \pm 0.52$ & $1.75 \pm 1.04$ & 0.3 (froz.) & $0.11 \pm 0.14$ & 1.02 & 0.43 & 0.05 & 0.11 & 0.02 \\
\hline \multicolumn{10}{|l|}{ Class II } \\
\hline $6^{*}$ & $3.36 \pm 1.60$ & $28.02 \pm 96.82$ & 0.30 (froz.) & $0.19 \pm 0.08$ & 0.77 & 0.73 & 0.18 & 0.20 & 0.15 \\
\hline $18^{\dagger}$ & $0.48 \pm 0.23$ & $3.04 \pm 1.46$ & 0.3 (froz.) & $0.09 \pm 0.06$ & 0.99 & 0.46 & 0.08 & 0.11 & 0.08 \\
\hline $23^{\dagger}$ & $0.7 \pm 0.28$ & $0.69 \pm 0.27$ & 0.3 (froz.) & $0.3 \pm 0.56$ & 1.08 & 0.37 & 0.07 & 0.31 & 0.01 \\
\hline 34 & $0.76 \pm 0.11$ & $3.22 \pm 0.5$ & 0.3 (froz.) & $0.78 \pm 0.21$ & 0.7 & 0.99 & 0.65 & 0.96 & 0 \\
\hline $44 \mathrm{f}$ & $1.41 \pm 0.34$ & $6.7 \pm 3.41$ & 0.3 (froz.) & $0.41 \pm 0.2$ & 0.73 & 0.91 & 0.43 & 0.62 & 0 \\
\hline $50^{\dagger}$ & $0.94 \pm 0.42$ & $3.43 \pm 2.42$ & 0.3 (froz.) & $0.25 \pm 0.24$ & 0.32 & 0.96 & 0.21 & 0.32 & 0.11 \\
\hline 54 & $3.16 \pm 0.11$ & $2.77 \pm 0.16$ & $0.15 \pm 0.06$ & $17.68 \pm 2.02$ & 1.08 & 0.17 & 7.12 & 19.2 & $0.6 e-2$ \\
\hline 59 & $1.5 \pm 0.21$ & $0.73 \pm 0.15$ & 0.3 (froz.) & $0.74 \pm 0.7$ & 0.65 & 0.87 & 0.08 & 0.76 & $0.6 e-3$ \\
\hline $63^{\dagger}$ & $6.17 \pm 3.04$ & $2.12 \pm 1.72$ & 0.3 (froz.) & $0.46 \pm 1.34$ & 0.8 & 0.57 & 0.1 & 0.48 & 0.05 \\
\hline 69 & $1.16 \pm 0.17$ & $1.02 \pm 0.13$ & 0.3 (froz.) & $0.69 \pm 0.37$ & 0.98 & 0.51 & 0.16 & 0.69 & $0.7 e-5$ \\
\hline 73 & $0.85 \pm 0.25$ & $1 \pm 0.19$ & 0.3 (froz.) & $0.42 \pm 0.23$ & 0.83 & 0.72 & 0.12 & 0.42 & $0.8 \mathrm{e}-3$ \\
\hline 75 & $0.52 \pm 0.14$ & $2.76 \pm 0.62$ & 0.3 (froz.) & $0.35 \pm 0.14$ & 0.65 & 0.98 & 0.29 & 0.41 & 0.02 \\
\hline $79^{\ddagger} \mathrm{f}$ & $0.52 \pm 0.04$ & $3.71 \pm 0.34$ & 0.3 (froz.) & $2.45 \pm 0.26$ & 1.00 & 0.50 & 2.44 & 3.16 & 0 \\
\hline \multicolumn{10}{|l|}{ Class II/III } \\
\hline 84 & $0.14 \pm 0.11$ & $1.24 \pm 0.11$ & 0.3 (froz.) & $0.13 \pm 0.07$ & 1.1 & 0.33 & 0.1 & 0.12 & 0.18 \\
\hline 85 & $0.29 \pm 0.28$ & $1.53 \pm 0.43$ & 0.3 (froz.) & $0.11 \pm 0.08$ & 1.11 & 0.34 & 0.07 & 0.1 & 0.32 \\
\hline \multicolumn{10}{|l|}{ Class III } \\
\hline 2 & $0.41 \pm 0.11$ & $1.32 \pm 0.16$ & $0.11 \pm 0.07$ & $0.71 \pm 0.36$ & 0.86 & 0.78 & 0.29 & 0.53 & $0.1 \mathrm{e}-4$ \\
\hline $3^{\dagger}$ & $0.03 \pm 0.22$ & $0.49 \pm 0.2$ & 0.3 (froz.) & $0.06 \pm 0.14$ & 0.54 & 0.85 & 0.06 & 0.05 & 0.26 \\
\hline 11 & $0.47 \pm 0.05$ & $1.69 \pm 0.15$ & $0.03 \pm 0.04$ & $2.65 \pm 0.72$ & 0.96 & 0.62 & 1.2 & 2.02 & 0.02 \\
\hline $21^{\dagger}$ & $0.9 \pm 0.34$ & $3.62 \pm 2.11$ & 0.3 (froz.) & $0.19 \pm 0.14$ & 1.15 & 0.29 & 0.16 & 0.24 & 0 \\
\hline 27 & $0.72 \pm 0.29$ & $1.1 \pm 0.3$ & $0.04 \pm 0.06$ & $0.82 \pm 0.96$ & 0.68 & 0.96 & 0.18 & 0.49 & 0.07 \\
\hline $29^{\dagger}$ & $1 \pm 0.26$ & $1.01 \pm 0.22$ & 0.3 (froz.) & $0.26 \pm 0.22$ & 0.48 & 0.94 & 0.07 & 0.26 & $0.4 \mathrm{e}-3$ \\
\hline 38 & $0.05 \pm 0.06$ & $2.53 \pm 0.5$ & $0.43 \pm 0.36$ & $0.26 \pm 0.13$ & 0.98 & 0.52 & 0.34 & 0.31 & $0.3 \mathrm{e}-6$ \\
\hline 45 & $1.05 \pm 0.20$ & $0.94 \pm 0.15$ & 0.30 (froz.) & $0.35 \pm 0.24$ & 0.85 & 0.65 & 0.08 & 0.37 & 0.09 \\
\hline 47 & $0.07 \pm 0.09$ & $0.63 \pm 0.06$ & 0.3 (froz.) & $0.14 \pm 0.09$ & 0.88 & 0.66 & 0.14 & 0.14 & 0.32 \\
\hline $57^{\dagger}$ & $3.45 \pm 1.33$ & $4.92 \pm 4.5$ & 0.3 (froz.) & $0.17 \pm 0.23$ & 0.56 & 0.85 & 0.12 & 0.25 & $0.9 \mathrm{e}-3$ \\
\hline 65 & $1 \pm 0.14$ & $2.95 \pm 0.51$ & 0.3 (froz.) & $0.91 \pm 0.28$ & 0.69 & 0.99 & 0.65 & 1.09 & 0 \\
\hline 67 & $0.84 \pm 0.14$ & $2.58 \pm 0.39$ & 0.3 (froz.) & $0.91 \pm 0.29$ & 0.75 & 0.95 & 0.61 & 1.03 & $0.5 \mathrm{e}-7$ \\
\hline \multicolumn{10}{|l|}{ Not a star } \\
\hline 70 & $1.1 \pm 0.52$ & $63.96 \pm 230.2$ & 0.3 (froz.) & $0.05 \pm 0.05$ & 0.83 & 0.77 & 0.35 & 0.41 & $0.1 \mathrm{e}-3$ \\
\hline
\end{tabular}

$\mathrm{f}$ - Source undergoing an intense flare. * - Unlikely a stellar source. ${ }^{\dagger}$ Spectral fit $\Delta E=0.1-7.5 \mathrm{keV}$. ${ }^{\ddagger}$ Spectral fit $\Delta E=0.3-8.5 \mathrm{keV}$.

In order to quantify the X-ray variability of these 19 YSOs we computed the normalized cumulative distribution of the amplitude of their variability. This represents the fraction of time that a source spends in a state with the flux larger than a given value, expressed in terms of a given normalization value, which can be the minimum count rate, the median count rate, etc. For the present sample we took as normalization value the count rate above which a source spends $90 \%$ of the time; this is less sensitive to noise fluctuations than the real minimum.

Figure 8 shows the distribution for the stars in our sample. Some sources (e.g. 2, 48 and 54) show mostly low-amplitude variability, in which less than $30 \%$ of the time is spent in a state 1.3-2 times above the minimum. Other sources (e.g. 21, 37, and 65 ) on the other hand, spend more than $50 \%$ of the time in such a state. Kolmogorov-Smirnov tests for the cumulative distributions of various pairs of sources also indicate the presence of different behaviors. However sources of the same class are present in groups with different variability behavior, so that an interpretation in terms of source evolution is not possible. This lack of trends in the variability of different classes of YSOs was also observed for a sample of WTTS and CTTS in L1551 (Giardino et al. 2006). 


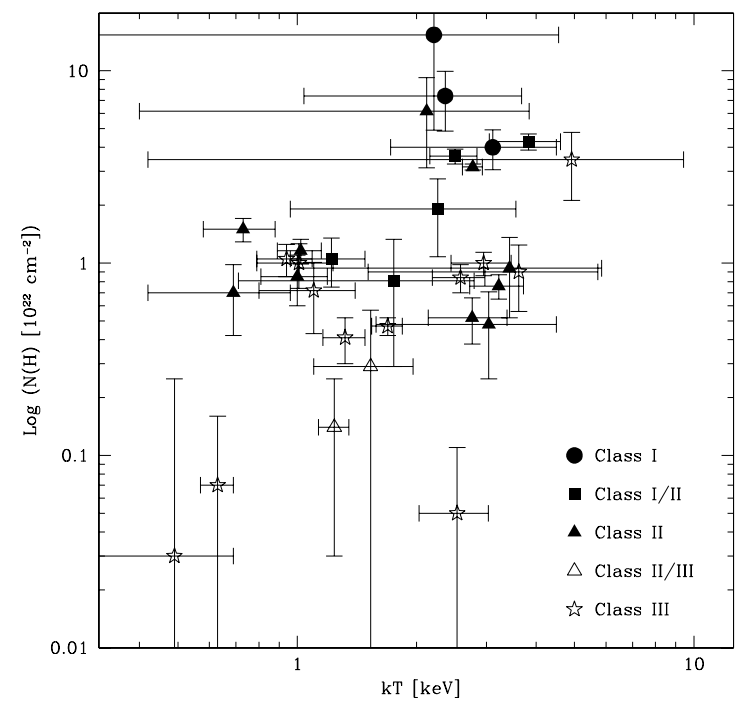

Fig. 7. Values of absorbing column densities vs plasma temperatures for the sources in Table 2. Sources undergoing a strong flare $(79,66,44)$ or likely non-stellar $(6,13$ and 70$)$ have been omitted. Error bars are at $1 \sigma$.

Table 3. Median values of absorbing column density, plasma temperature, and X-ray luminosity for the different classes. Units as from Table 2. Flaring sources and sources likely non-stellar are not included.

\begin{tabular}{l|ccc}
\hline \hline Class & $N(\mathrm{H})$ & $k T$ & $L_{\mathrm{X}}$ \\
\hline & $N_{22}$ & $\mathrm{keV}$ & $L_{30}$ \\
\hline I & 7.4 & 2.4 & 1.7 \\
I/II & 1.9 & 2.3 & 0.3 \\
II & 0.9 & 2.8 & 0.4 \\
II/III & 0.3 & 1.5 & 0.1 \\
III & 0.8 & 1.7 & 0.2 \\
\hline I/II + II & 1.0 & 2.3 & 0.3 \\
II/III + III & 0.7 & 1.5 & 0.2 \\
\hline
\end{tabular}

\section{Interesting individual sources}

As already mentioned, we extracted the light curves and spectra of all the sources with count rate $\geq 2 \mathrm{cts} / \mathrm{ks}$. These are shown in Fig. 10 and Fig. 13 and the results of the spectral fits for these sources are summarised in Table 2. In this section we comment on those sources which have a peculiarity or show an interesting behavior.

\subsection{Source 13}

A relatively bright X-ray source, it has no 2MASS counterpart but it has a Spitzer counterpart with colors apparently compatible with a Flat Spectrum YSO. Its X-ray spectrum is however very hard and strongly absorbed. If it were a thermal spectrum, the temperature would be in excess of $20 \mathrm{keV}$. A $\alpha=1.8$ power law provides as good a fit as the thermal model; in both cases a significant soft excess remains. The absorbing column density is 3 to $4 \times 10^{22} \mathrm{~cm}^{-2}$, depending on the spectral model used. Such spectrum is very unlikely to originate in a stellar source, and therefore we consider this source most likely an active background extragalactic object (an AGN). The source is present in the XMM-Newton observation of Preibisch (2003), who identified an IR counterpart (but does not extract an X-ray spectrum).

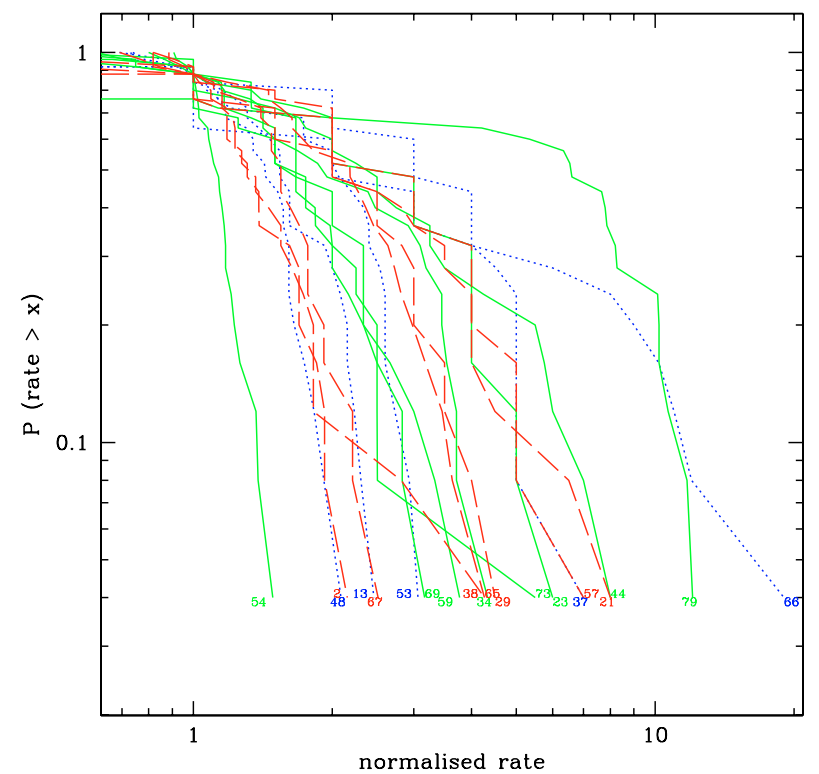

Fig. 8. Normalized cumulative count-rate distribution for all the YSOs in Table 2 with Kolmogorov-Smirnov probability of constancy $\left(C_{\mathrm{KS}}\right)$ lower than 2\%. Dotted line indicates Class I/II objects, continuous line Class II, and short-dashed Class III objects.

\subsection{Source 44}

Classified as a Class II source, on the basis of the ISO and its Spitzer colors, the source is observed during an intense (a factor of $\simeq 4$ ) flare, as reflected in the high X-ray temperature, $k T=6.7 \mathrm{keV}$. The large absorption $N(\mathrm{H})=1.4 \times 10^{22} \mathrm{~cm}^{-2}$ $\left(A_{\mathrm{V}}=7.3\right)$ points to a source deep in the cloud. The source was first detected at infrared wavelengths by Eiroa \& Casali (1989) and Eiroa \& Casali (1992) and later observed with the VLA (Bontemps 1996).

\subsection{Source 48}

Source 48 is the X-ray counterpart of SVS 20, the deeply embedded IR double source located inside the "eye" structure at the center of the Serpens nebula. Eiroa et al. (1987) derived a separation of 1.6 arcsec between the two components and estimated a total luminosity in the range $7-45 L_{\odot}$; Ciardi et al. (2005) found that the temperatures and luminosities of the two protostellar objects are quite different, for SVS-S they derived $L \approx 20-80 L_{\odot}$ and for SVS-N $L \approx 0.9 L_{\odot}$. Doppmann et al. (2005) estimated for SVS-S a luminosity of $24.7 L_{\odot}$. As can be seen from Fig. 9, in the Chandra image the PSF of this source is elongated in the North-East direction and PwDETECT places three sources (48, 49 and 51) within it. At about 3 arcmin off-axis the two components of SVS 20, cannot be fully resolved by Chandra. Source 48 and 49 , however, are at the right distance and orientation to be the X-ray counterparts of SVS 20-S and N respectively. The presence of emission to the NE of Source 49 is unexpected and points to a more complex situation, perhaps the presence of a third source (without IR counterpart) or of an X-ray emitting jet. Using near-IR polarimetry combined with narrow-band imaging of molecular hydrogen emission, Huard et al. (1997) detected outflow activity in the surroundings of SVS 20, and in particular they observed a knot of emission at $\sim 20^{\prime \prime}$ distance from SVS 20 , in the NE direction. 
North

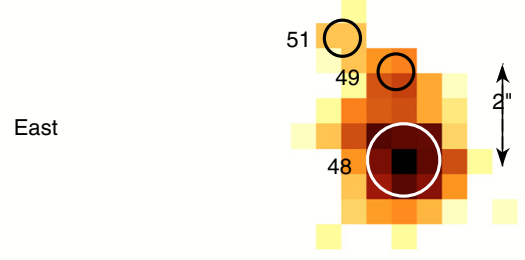

Fig. 9. X-ray image of SVS 20 (source 48). Source 48 and 49 are at the right distance and orientation to be the X-ray counterpart of SVS 20-S and SVS20-N respectively. Source 51 could be a very absorbed stellar source or a background AGN (see text).

We extracted the photons from a small $\left(\sim 1^{\prime \prime}\right.$ radius $)$ region around source 51 and obtained roughly 20 photons with a median energy of $\simeq 5 \mathrm{keV}$. This is inconsistent with $\mathrm{X}$-ray emission from a stellar outflow whose typical energy are below $1 \mathrm{keV}$ and would point to a very absorbed stellar source or an extragalactic background source. The source, however, is not fully resolved from sources 49 and 48 , which will likely contaminate it (more so at high energies, because of the energy dependence of the PSF), so that one cannot draw definite conclusions on the spectral energy distribution of this source.

ISO and Spitzer colors for the unresolved system indicate a Flat Spectrum source. In the X-ray the star shows significant variability, with variations of a factor of two on a $20 \mathrm{ks}$ time scale. With $k T=2.5 \mathrm{keV}$ and $N(\mathrm{H})=3.6 \times 10^{22} \mathrm{~cm}^{-2}$, equivalent to $A_{V}=19 \mathrm{mag}$, the source is relatively hot and deeply embedded in the cloud.

\subsection{Source $54-$ [EC92] 95}

[EC92] 95 is a heavily absorbed intermediate-mass $\left(\sim 4 M_{\odot}\right)$ YSO, for which Preibisch (1999) derived, from IR spectroscopy, a spectral type $\mathrm{K} 2$, with $A_{\mathrm{V}} \sim 36 \mathrm{mag}$ and $L_{\mathrm{bol}} \sim 60 L_{\odot}$. Assuming that the X-ray absorption was the same as the optical one, Preibisch (1998) attributed an extremely large X-ray luminosity to this $\operatorname{star}\left(L_{\mathrm{X}} \sim 10^{33}\right)$, much higher than any known stellar source. XMM-Newton observations (Preibisch 2003) allowed the X-ray absorption to be directly determined, showing it to be over a factor of two lower than the optical estimate, thus bringing the X-ray luminosity to a value within the range observed in YSOs. The reason why the two absorption determinations (optical and X-ray) differ is, however, still unknown.

The X-ray spectral parameters determined from this Chandra observation are $k T=2.7 \pm 0.2 \mathrm{keV}$ and $L_{\mathrm{X}}=$ $1.9 \times 10^{31} \mathrm{erg} \mathrm{s}^{-1}$, with $N_{\mathrm{H}}=(3.2 \pm 0.1) \times 10^{22} \mathrm{~cm}^{-2}$ and $Z=0.2 Z_{\odot}$, remarkably consistent with the parameters derived by Preibisch (2003) for the XMM-Newton observation, showing that the source has little long-term variability in its X-ray emission.

Preibisch (2003) speculated about a number of possible scenarios for the discrepancy, including some unidentified peculiarity of flat-spectrum YSOs, by analogy with IRS 5 in L1551. However, the line of reasoning of Preibisch (2003) is based on the assumption that X-ray emission is observed from the IRS 5 protostar (which has $A_{\mathrm{V}} \sim 150 \mathrm{mag}$ ). As demonstrated by Favata et al. (2002) and Bally et al. (2003) the X-ray emission in this case comes from the associated Herbig-Haro object $\mathrm{HH}$ 154, which has, given that the jet penetrates through the absorbing cloud, a much lower absorption, with $A_{\mathrm{V}} \sim 7 \mathrm{mag}$, compatible with the absorption measured for the X-ray source.

One possible explanation for the peculiar discrepancy between the X-ray and optically determined absorption columns is that the source is surrounded by a thick accreting disk, and is seen nearly edge-on (explaining the large optically determined $A_{\mathrm{V}}$ ). If at the same time the $\mathrm{X}$-ray emission comes from a region somewhat displaced above or below the disk (as it could be the case for a large polar corona), this could explain the lower absorbing column density observed in X-rays. However, this implies a corona with a significant scale height above the photosphere, and this is in conflict with recent evidence, for example, with the observed occurrence of rotational modulation in YSOs in Orion (Flaccomio et al. 2005), which implys that most coronal material is located in compact structures. While large flaring magnetic loops have been observed, also in Orion YSOs (Favata et al. 2005a), the consistency of the X-ray emission from [EC92] 95 over a number of years appears to rule out the hypothesis that one may be seeing a large flaring loop extending at a large distance from the star's photosphere. Indeed, if the observed source variability (Fig. 10), is due to a flare, then the timescales of such variability ( $\$ 20 \mathrm{ks})$ would imply a relatively compact structure $\left(L_{\text {loop }} \lesssim 5 R_{\odot}\right)$.

Another possible explanation for the discrepancy between the X-ray and optically determined absorption columns would be the presence of a lower mass companion. This companion star would have to be displaced from the disk of the main star, and be the main source of X-ray luminosity while contributing only a small fraction of the optical flux. Nevertheless, for such a companion star to have an X-ray luminosity of $1.9 \times 10^{31} \mathrm{erg} \mathrm{s}^{-1}$, its mass should be around 2-3 $M_{\odot}$, as can be inferred by looking at the scatter plot of X-ray luminosity versus star mass in e.g. Flaccomio et al. (2003) and therefore would provide significant contribution to the luminosity of the system.

\subsection{Source 57}

This source lies at 3.7 arcsec from the position of the HerbigHaro flow HH456 identified by Davis et al. (1999). We believe it unlikely that the X-ray emission originates from shocks generated by the Herbig-Haro flow (as reported by e.g. Pravdo et al. (2001) for HH 2 or Favata et al. (2002) for HH 154). The source is weak so its spectral characteristics cannot be well constrained, however it appears to be harder than generally observed in Herbig-Haro flows.

The source has a 2MASS counterpart at only 0.2 arcsec distance and corresponding Spitzer source classified as a Class III star. A spectral fit with an absorbed $1 \mathrm{~T}$ plasma gives $N(\mathrm{H})=$ $(3.5 \pm 1.3) \times 10^{22} \mathrm{~cm}^{-2}$ and $k T=4.9 \pm 4.5(P=0.85)$, consistent with the X-ray emission from the stellar corona of a CTTS or WTTS star.

\subsection{Source 70}

This source has no 2MASS nor optical counterpart. It has no counterpart within the ISO catalogue by Kaas et al. (2004) and it was detected by Spitzer only in three of the IRAC channels $(4.5,5.8$, and $8.0 \mu \mathrm{m})$, so the source could not be classified. Its $\mathrm{X}$-ray spectrum is very hard. A fit with a thermal spectrum gives 

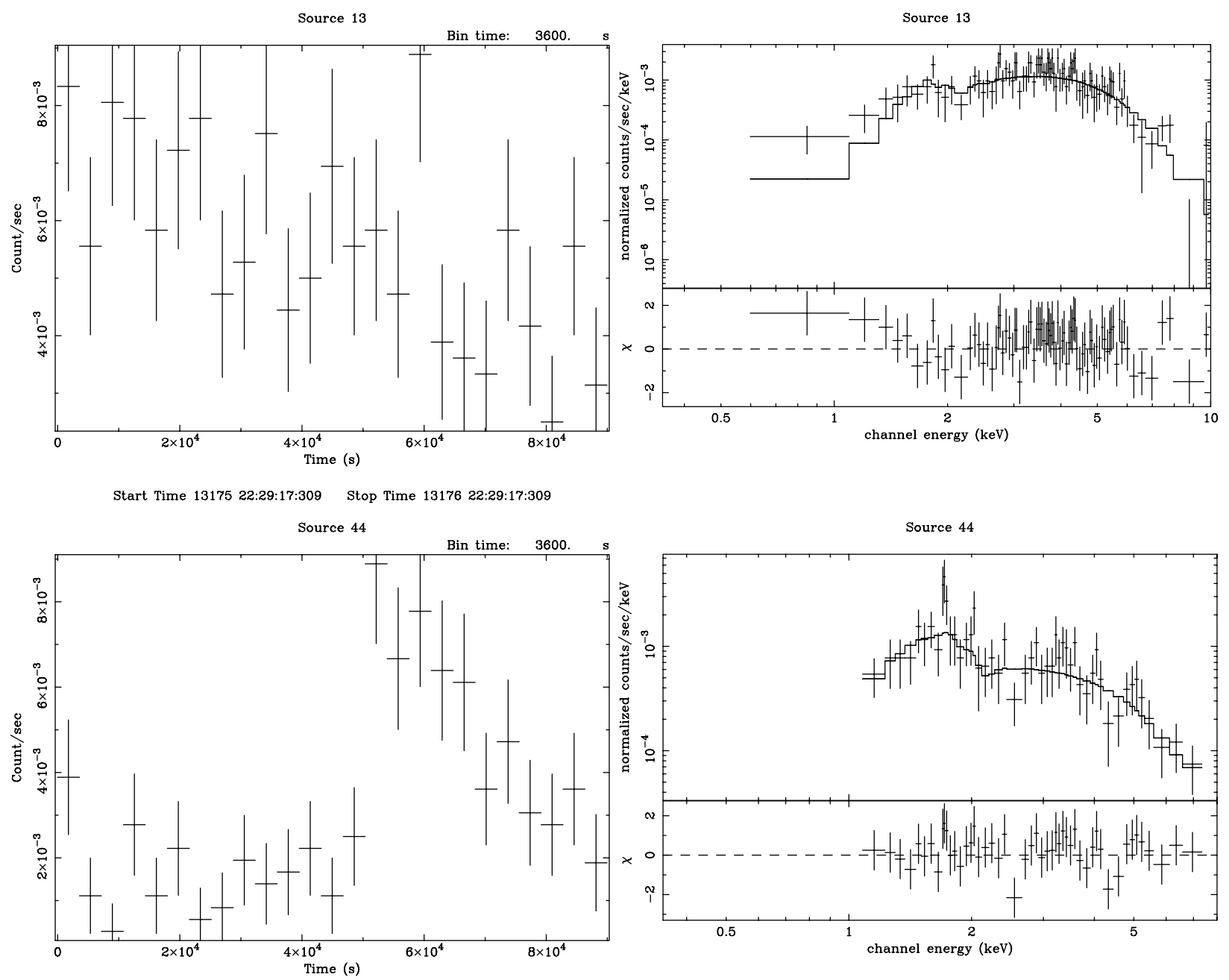

Start Time 13175 22:29:17:309 Stop Time 13176 22:29:17:309
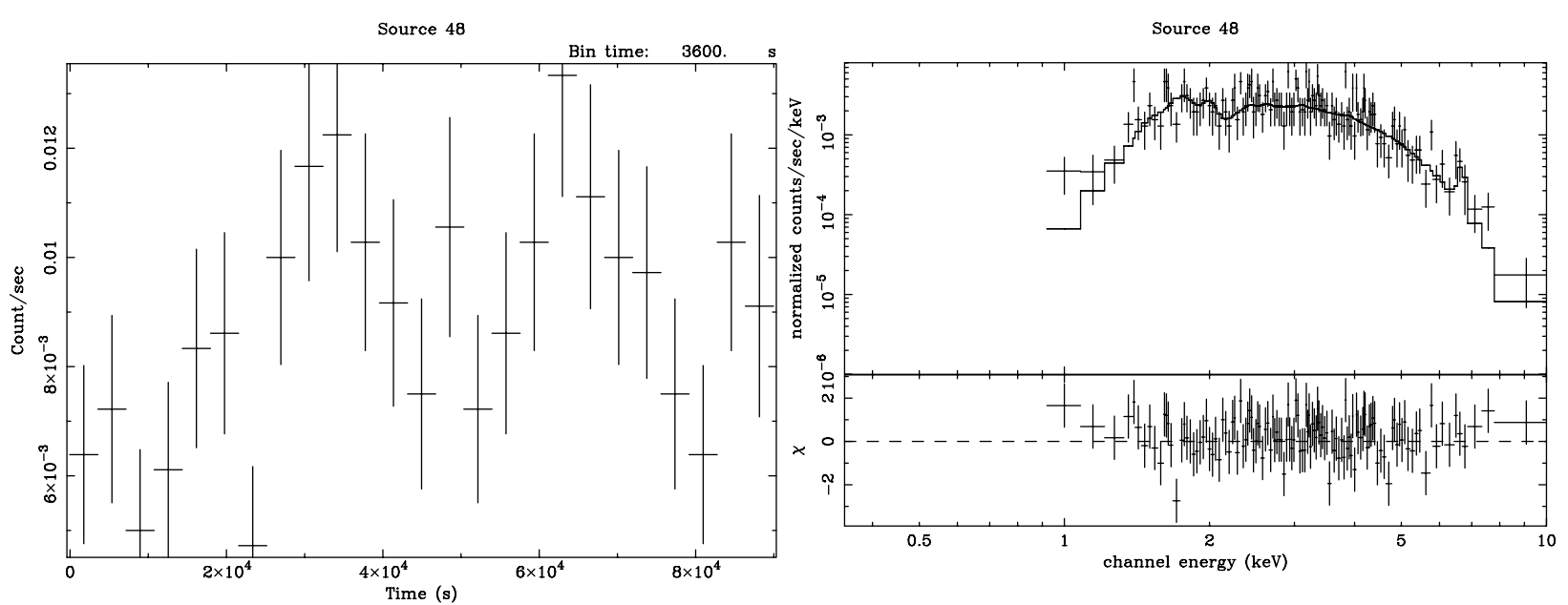

Start Time 13175 22:29:17:309 Stop Time 13176 22:29:17:309

Fig. 10. Light curves and spectra with spectral fits of sources discussed in the text. See Sect. 4.

a temperature of $60 \mathrm{keV}$, but it is badly constrained. A $\alpha=1.2$ power law provides as good a fit as the thermal model. Such a spectrum is unlikely to originate in a stellar source, and therefore we consider this most likely an X-ray bright background extragalactic object. The source is present in the XMM-Newton observation of Preibisch (2003), who also does not detect any near IR counterpart.

\subsection{Source 79}

During our observation, this Class II object, identified both in the ISO and Spitzer data, undergoes a strong, long-duration, flare: the intensity of the source increases by a factor of 10 , impulsively, and then slowly decays over a period of more than $50 \mathrm{ks}$. To determine the spectral parameters of the flaring emission we 

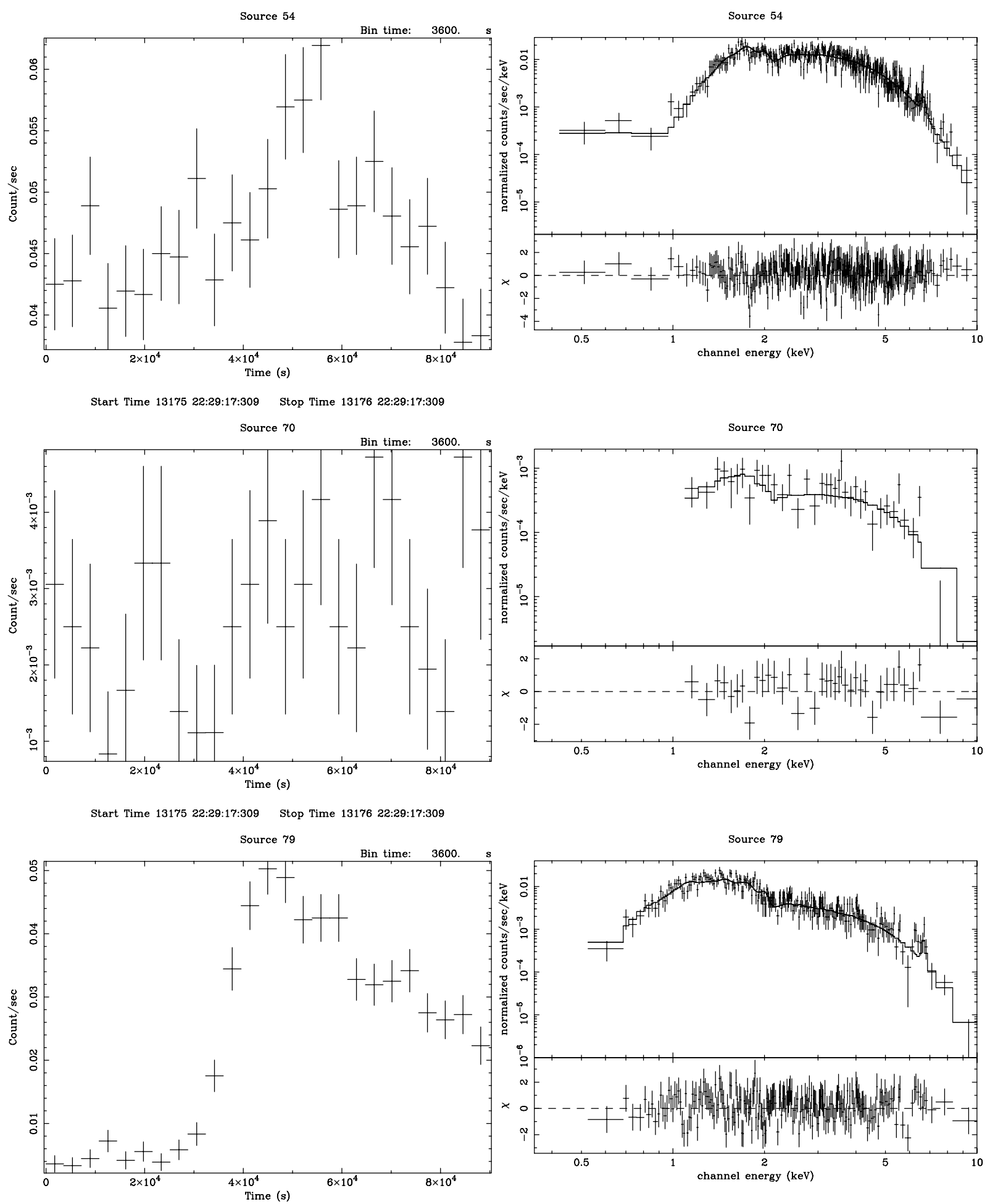

Start Time 13175 22:29:17:309 Stop Time 13176 22:29:17:309

Fig. 10. continued.

subdivided the data for this source in four time intervals: the quiescent phase, the rise phase of the flare, its peak, and the flare decay. The parameters of the spectral fits derived for these four time intervals are summarised in Table 4.

To derive the flare's physical parameters we used the approach initially discussed by Reale et al. (1997) and since then applied to a variety of stellar flares. This approach uses the slope $\zeta$ of the flare decay in the $\log T$ versus $\log \sqrt{E M}$ diagram to account properly for the presence of sustained heating during the flare decay. The calibration of the method for Chandra ACIS, and a detailed explanation of the physics behind it, can be found in Favata et al. (2005a), to which the reader is referred. In this formulation, the semi-length of the flaring loop is given by

$L=\frac{\tau_{\mathrm{LC}} \sqrt{T_{\max }}}{\alpha F(\zeta)} \quad 0.32<\zeta \lesssim 1.5$ 
Table 4. Best-fit spectral parameters for the quiescent phase and flaring phases of source 79; units are $N_{22}=10^{22} \mathrm{~cm}^{-2}$ and $E M_{53}=10^{53} \mathrm{~cm}^{-3}$. Metal abundance was fixed at $Z=0.3 Z_{\odot}$.

\begin{tabular}{r|cccccc}
\hline \hline Phase & $N(\mathrm{H})$ & $k T$ & $E M$ & $\chi^{2}$ & $P$ & Rate \\
\hline & $N_{22}$ & $\mathrm{keV}$ & $E M_{53}$ & & & $\mathrm{cts} / \mathrm{s}$ \\
\hline Quiesc. & $0.98 \pm 0.22$ & $0.96 \pm 0.18$ & $0.92 \pm 0.69$ & 1.32 & 0.16 & $4.2 \pm 0.4$ \\
Rise & $0.61 \pm 0.20$ & $5.45 \pm 3.12$ & $1.65 \pm 0.65$ & 0.97 & 0.52 & $17.6 \pm 1.2$ \\
Peak & $0.60 \pm 0.10$ & $4.79 \pm 1.22$ & $4.95 \pm 1.13$ & 0.80 & 0.90 & $49.3 \pm 2.2$ \\
Decay & $0.44 \pm 0.05$ & $3.42 \pm 0.31$ & $3.31 \pm 0.45$ & 0.93 & 0.72 & $32.9 \pm 0.9$ \\
\hline
\end{tabular}

where $\alpha=3.7 \times 10^{-4} \mathrm{~cm}^{-1} \mathrm{~s}^{-1} \mathrm{~K}^{1 / 2}, \tau_{\mathrm{LC}}$ is the $1 / e$ folding time of the light curve decay, and $T_{\max }$ is the peak temperature of the plasma in the flaring loop. The limits of applicability of Eq. (1) correspond, on one side ( $\zeta \simeq 1.5$ ), to a freely decaying loop, with no sustained heating, on the other $(\zeta=0.32)$, to a sequence of quasi-static states for the loop, in which the heating timescale is so long as to mask the loop's intrinsic decay. $F(\zeta)$ and the relationship between $T_{\max }$ and the best-fit peak temperature $T_{\text {obs }}$ are both functions that need to be separately determined for each $\mathrm{X}$-ray detector, depending on its spectral response. For ACIS,

$F(\zeta)=\frac{0.63}{\zeta-0.32}+1.41$

and

$T_{\max }=0.068 T_{\mathrm{obs}}^{1.20}$.

The $\log T$ versus $\log \sqrt{E M}$ diagram for the flare of source 79 is shown in Fig. 11. The lowest point refers to the quiescent phase, the highest point corresponds to the rise phase of the flare, and the line joins the points relatives to the flare peak and its decay. The slope of this line is $\zeta=1.67$, which is outside the limit of applicability of Eq. (1), however the error bars of the points are compatible with $\zeta=1.0-1.5$. Given a flare decay $1 / e$-folding time $\tau_{\mathrm{LC}}=53 \mathrm{ks}$, Eqs. (2) and (1) result in a loop semi-length of $L=10-12 R_{\odot}$.

Source 79 appears to be a very-low-mass star; its position in the color-magnitude diagram of Fig. 2 implyies a mass $M<0.1 M_{\odot}$. According to the evolutionary model of Siess et al. (2000), at 2 Myr of age, such a star would have a radius $R<R_{\odot}$, hence, the flaring loop of source 79 extends to a dinstance of more than 10 times the stellar radius. Assuming for the loop a radius $r=0.1 L$, typical of solar events, from the emission measure at the flare maximum, one derives an electron density $n_{\mathrm{e}} \simeq 3.6 \times 10^{9} \mathrm{~cm}^{-3}$ and a corresponding equipartition magnetic field strength $B \simeq 60 \mathrm{G}$.

Very long flaring structures in YSOs have been recently reported for half a dozen sources in the ONC by Favata et al. (2005a) and, more tentatively, for HL Tau in L1551 by Giardino et al. (2006). The flare in source 79 is similar, even though the star is significantly less massive than HL Tau and the objects in the ONC that exhibited evidence of very long flaring structures. This indicates that these long flaring structures develop in stars across a wide mass range (from $M_{\star}$ apparently well below $0.1 M_{\odot}$ to $M_{\star} \sim 2.4 M_{\odot}$, the most massive object in the COUP flaring sample).

As the majority of YSOs is surrounded by disks, Favata et al. (2005a) speculated that the large magnetic structures that confine the flaring plasma are actually the same type of structures that connect the star to the circumstellar disk, in the magnetospheric

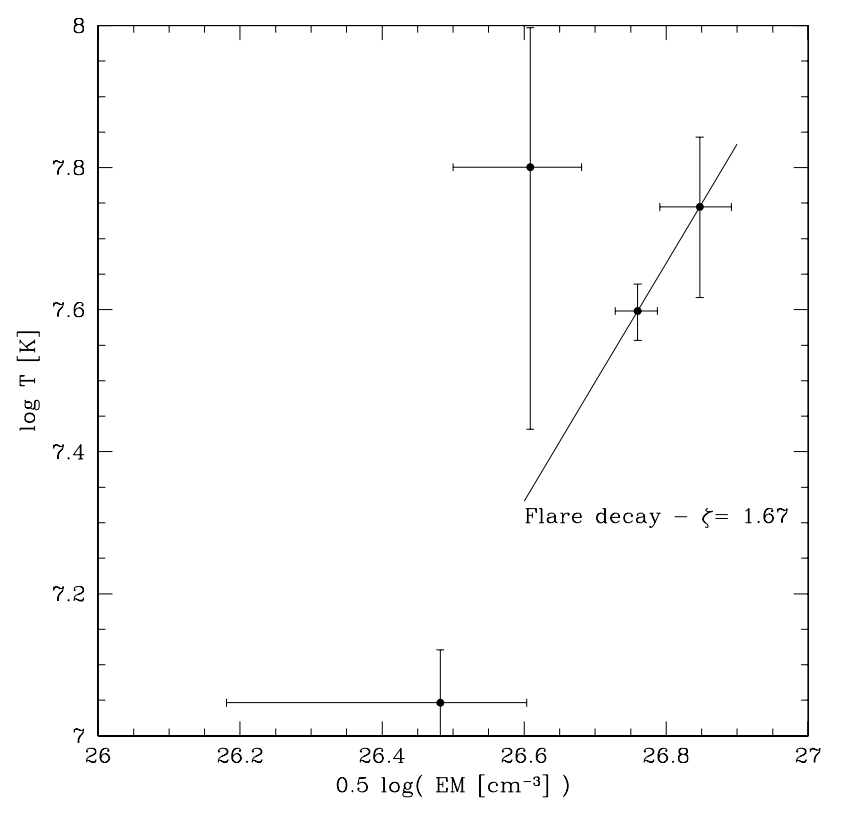

Fig. 11. The evolution of the flare of source 79 in the $\log T$ vs. $\log \sqrt{E M}$ plane.

accretion paradigm. As described below, we detect, in the spectrum of this star, $6.4 \mathrm{keV} \mathrm{Fe}$ fluorescent emission with a large equivalent width, compatible with it being due to fluorescence from a centrally irradiated circumstellar disk.

The spectrum (integrated over the entire observation) of source 79 is shown in Fig. 10 together with its spectral fit with an absorbed 1T plasma model. There appears to be an excess of emission redward of the $\mathrm{Fe} \mathrm{K}$ complex at $\simeq 6.7 \mathrm{keV}$, at the expected energy of the $6.4 \mathrm{keV}$ Fe fluorescent line. To determine the significance of this excess, we fitted the spectrum of source 79 again with an additional Gaussian line component at $6.4 \mathrm{keV}$, which was constrained to be narrow $(10 \mathrm{eV})$, while its normalisation was unconstrained. The other parameters of the absorbed 1T plasma model (absorbing column density, temperature and normalisation of the thermal spectrum) were also unconstrained. The result of this new fit can be compared with the original fit in Fig. 12: the excess at $6.4 \mathrm{keV}$ is well accounted for by a narrow Gaussian line with fitted intensity $I=(3.79 \pm 5.78) \times 10^{-7}$ photons $\mathrm{cm}^{-2} \mathrm{~s}^{-1}$. The fitted values of the absorbed $1 \mathrm{~T}$ plasma model do not change significantly with the addition of the Gaussian line at $6.4 \mathrm{keV}$ (cf. Table 2), while the null-hypothesis probability of the fit increases marginally from $50 \%$ to $54 \%$. 

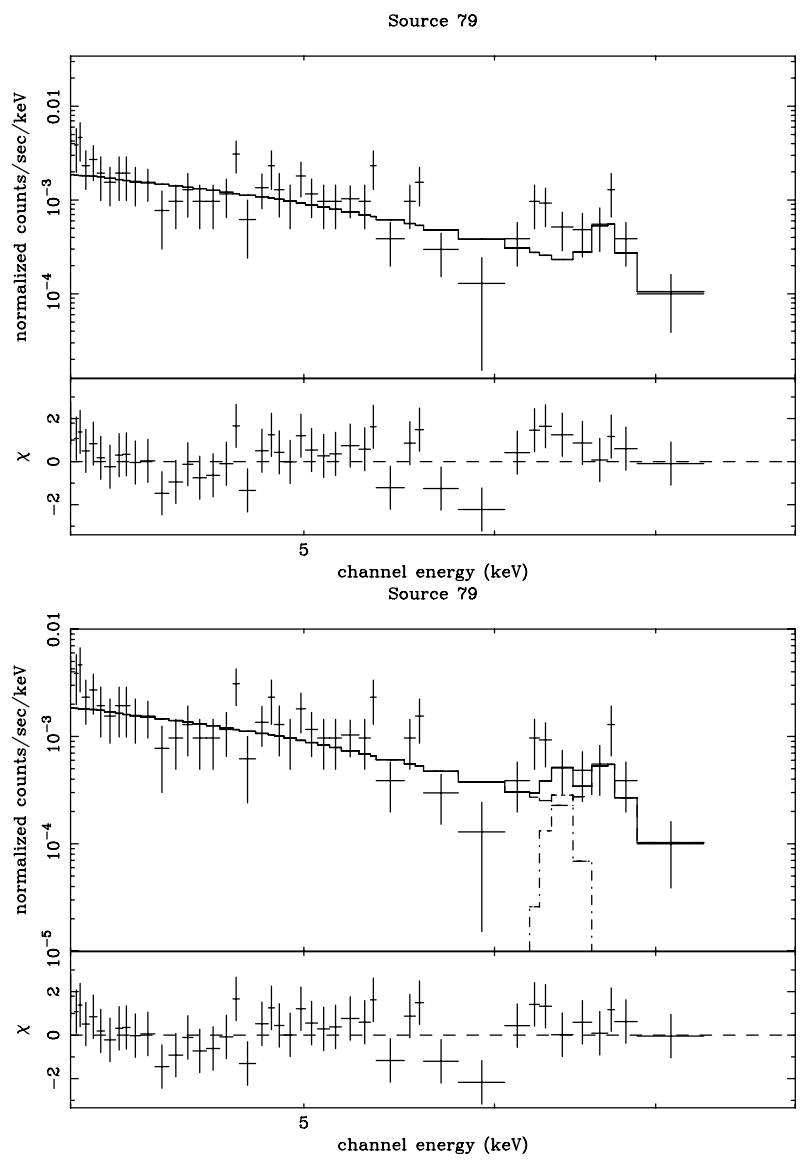

Fig. 12. Spectrum of source 79 in the $4.0-8.0 \mathrm{keV}$ region and spectral fit using an absorbed thermal model (top) and with the addition of a narrow Gaussian component (bottom) at $6.4 \mathrm{keV}$ (Fe fluorescent line).

The equivalenth width of the fitted line is $W_{\alpha}=149 \mathrm{eV}^{5}$. As discussed in Favata et al. (2005b), such a large equivalenth width cannot be explained by fluorescent emission from colder, diffuse (optically thin) circumstellar material: the absorbing column density required would in fact be of the order of $10^{24} \mathrm{~cm}^{-2}$ in conflict with the value of $0.5 \times 10^{22} \mathrm{~cm}^{-2}$ derived from the spectral fit. Under optically thick conditions, the computation of the equivalent width of the fluorescence line requires a detailed radiative transfer treatment and the assumption of a well defined geometry. Using the computation by George \& Fabian (1991), for a spectral index $\gamma=2-2.3$, a centrally irradiated accretion disk (viewed face-on) would entail $W_{\alpha}=120-150 \mathrm{eV}$. The X-ray spectrum of source 79 is similar to the model spectra that were used by George \& Fabian $(1991)^{6}$, hence, the equivalent width of the $6.4 \mathrm{keV}$ line is compatible with Fe fluorescent emission from a centrally irradiated disk.

\section{Conclusions}

Our $90 \mathrm{ks}$ Chandra observations of the Serpens Cloud Core allowed the X-ray emission from this embedded young star

\footnotetext{
${ }^{5}$ In computing the line equivalent width XSPEC does not provide an error, as this would involve an estimate of the error of the fitted continuum. We note, however, that given the large uncertainties in the fitted line intensity the uncertainties on $W_{\alpha}$ are also large: i.e. of the order of $150 \%$.

6 The data of source 79 can also be fitted $(P=0.60)$ by an absorbed power law with spectral index $\gamma=2.3 \pm 0.09$.
}

cluster to be studied at high sensitivity. One of the goals of the observation was to search for X-ray emission from confirmed, bona fide Class 0 sources, a still elusive target. We did not detect X-ray emission from any of the six well studied Class 0 objects in the cloud core. The sensitivity of our observation allows us to put an upper limit to the X-ray emission from these Class 0 sources: assuming that the absorbing column density is $\lesssim 4 \times 10^{23} \mathrm{~cm}^{-2}\left(A_{\mathrm{V}} \lesssim 200 \mathrm{mag}\right.$ ), and that the coronal temperature is $k T \simeq 2.5 \mathrm{keV}$ (as observed in the Class I sources of Serpens), the typical X-ray luminosity of Class 0 sources must be $L_{X} \lesssim$ $0.4 \times 10^{30} \mathrm{erg} \mathrm{s}^{-1}$. If one assumes a higher plasma temperature of $3.5 \mathrm{keV}$ - the average plasma temperature of Class I sources in $\rho$ Oph (Ozawa et al. 2005) - then the typical luminosity of Class 0 sources must be $L_{\mathrm{X}} \lesssim 0.1 \times 10^{30} \mathrm{erg} \mathrm{s}^{-1}$ or the absorbing column densities must be even higher. Class I sources in this region and $\rho$ Oph have typical luminosities $L_{\mathrm{X}} \gtrsim 1 \times 10^{30} \mathrm{erg} \mathrm{s}^{-1}$.

We performed a spectral and timing analysis of all the sufficiently X-ray bright YSOs. Our data show a clear pattern of increasing column densities from Class III to Class I sources, and also hint at evolution in the X-ray emitting plasma temperature of these YSOs. No evolutionary trend is found in these sources' temporal variability.

During our observations the low-mass Class II source, source 79, undergoes a large, long-duration, flare for which we derive a semi-loop length of $L=10-12 R_{\odot}$. Such a long flaring loop $(\sim 0.1 \mathrm{AU})$ could be explained if the flare is due to a magnetic reconnection event of a flux tube linking the star's photosphere with the inner rim of a circumstellar disk, as in the magnetospheric accretion model. Interestingly, we detect, in the spectrum of this star, $6.4 \mathrm{keV} \mathrm{Fe}$ fluorescent emission with a large equivalent width, compatible with reflection off a circumstellar disk irradiated by the hard X-ray continuum emission of the flare.

Acknowledgements. We thank Ettore Flaccomio for his help in producing the coadded image of Class 0 sources, Andrew Thean for helping us improve the language of the paper, and Olive Buggy for her prompt help with software installation problems. G.M. and S.S. acknowledge financial contribution from contract ASI-INAF I/023/05/0. This research makes use of data products from the Two Micron All Sky Survey, which is a joint project of the University of Massachusetts and the Infrared Processing and Analysis Center/California Institute of Technology, funded by the National Aeronautics and Space Administration and the National Science Foundation.

\section{References}

Andre, P., Ward-Thompson, D., \& Barsony, M. 2000, Protostars and Planets IV, 59

Bahcall, J. N., \& Soneira, R. M. 1980, ApJS, 44, 73

Bally, J., \& Lada, C. J. 1983, ApJ, 265, 824

Bally, J., Feigelson, E., \& Reipurth, B. 2003, ApJ, 584, 843

Bontemps, S. 1996, Ph.D. Thesis

Casali, M. M., Eiroa, C., \& Duncan, W. D. 1993, A\&A, 275, 195

Churchwell, E., \& Koornneef, J. 1986, ApJ, 300, 729

Ciardi, D. R., Telesco, C. M., Packham, C., et al. 2005, ApJ, 629, 897

Davis, C. J., Matthews, H. E., Ray, T. P., et al. 1999, MNRAS, 309, 141

Doppmann, G. W., Greene, T. P., Covey, K. R., et al. 2005, AJ, 130, 1145

Eiroa, C., \& Casali, M. M. 1989, A\&A, 223, L17

Eiroa, C., \& Casali, M. M. 1992, A\&A, 262, 468

Eiroa, C., Lenzen, R., Leinert, C., et al. 1987, A\&A, 179, 171

Eiroa, C., Torrelles, J. M., Curiel, S., et al. 2005, AJ, 130, 643

Favata, F., Fridlund, C. V. M., Micela, G., et al. 2002, A\&A, 386, 204

Favata, F., Flaccomio, E., Reale, F., et al. 2005a, ApJS, 160, 469

Favata, F., Micela, G., Silva, B., et al. 2005b, A\&A, 433, 1047

Fazio, G. G., Hora, J. L., Allen, L. E., et al. 2004, ApJS, 154, 10

Flaccomio, E., Damiani, F., Micela, G., et al. 2003, ApJ, 582, 398

Flaccomio, E., Micela, G., Sciortino, S., et al. 2005, A\&A, submitted

Flaccomio, E., Micela, G., \& Sciortino, S. 2006, A\&A, 455, 903 
Forbrich, J., Preibisch, T., \& Menten, K. M. 2006, A\&A, 446, 155

George, I. M., \& Fabian, A. C. 1991, MNRAS, 249, 352

Getman, K. V., Feigelson, E. D., Gordon, G., et al. 2006, ApJ, Submitted [arXiv: astro-ph/0607006], 503

Giardino, G., Favata F., Silva, B., et al. 2006, A\&A, 453, 241

Giovannetti, P., Caux, E., Nadeau, D., et al. 1998, A\&A, 330, 990

Hamaguchi, K., Corcoran, M. F., Petre, R., et al. 2005, ApJ, 623, 291

Herbst, T. M., Beckwith, S. V. W., \& Robberto, M. 1997, ApJ, 486, L59

Hogerheijde, M. R., van Dishoeck, E. F., Salverda, J. M., et al. 1999, ApJ, 513, 350

Huard, T. L., Weintraub, D. A., \& Kastner, J. H. 1997, MNRAS, 290, 598

Hurt, R. L., \& Barsony, M. 1996, ApJ, 460, L45

Kaas, A. A., Olofsson, G., Bontemps, S., et al. 2004, A\&A, 421, 623

Motte, F., Andre, P., \& Neri, R. 1998, A\&A, 336, 150

Natta, A., Testi, L., \& Randich, S. 2006, A\&A, in press, [astro-ph/0602618], 245

Ozawa, H., Grosso, N., \& Montmerle, T. 2005, A\&A, 438, 661

Pravdo, S. H., Feigelson, E. D., Garmire, G., et al. 2001, Nature, 413, 708
Preibisch, T. 1998, A\&A, 338, L25

Preibisch, T. 1999, A\&A, 345, 583

Preibisch, T. 2003, A\&A, 410, 951

Preibisch, T. 2004, A\&A, 428, 569

Preibisch, T., Kim, Y.-C., Favata, F., et al. 2005, ApJS, 160, 401

Reale, F., Betta, R., Peres, G., et al. 1997, A\&A, 325, 782

Rieke, G. H., \& Lebofsky, M. J. 1985, ApJ, 288, 618

Rieke, G. H., Young, E. T., Engelbracht, C. W., et al. 2004, ApJS, 154, 25

Siess, L., Dufour, E., \& Forestini, M. 2000, A\&A, 358, 593

Sogawa, H., Tamura, M., Gatley, I., et al. 1997, AJ, 113, 1057

Straizys, V., Cernis, K., \& Bartasiute, S. 1996, Baltic Astron., 5, 125

Strom, S. E., Vrba, F. J., \& Strom, K. M. 1976, AJ, 81, 638

Testi, L., \& Sargent, A. I. 1998, ApJ, 508, L91

Tsuboi, Y., Koyama, K., Hamaguchi, K., et al. 2001, ApJ, 554, 734

Tsujimoto, M., Koyama, K., Kobayashi, N., et al. 2004, PASJ, 56, 341

White, G. J., Casali, M. M., \& Eiroa, C. 1995, A\&A, 298, 594

Winston, E. M., Megeath, S. T., Wolk, S., et al. 2006, ApJ, Submitted

Wolf-Chase, G. A., Barsony, M., Wootten, H. A., et al. 1998, ApJ, 501, L193 
G. Giardino et al.: The onset of X-ray emission in young stellar objects, Online Material $p 1$

\section{Online Material}




\section{Notes on individual sources}

We extracted the light curves and spectra of all the sources with count rate $\geq 2 \mathrm{cts} / \mathrm{ks}$. These are shown in Figs. 10 and 13 and the results of the spectral fits for these sources are summarised in Table 2. Below we comment on the brighter sources that were not discussed in Sect. 4.

\subsection{Source 2}

This X-ray source, already detected by Preibisch (2003), has an optical and (bright) IR counterpart and it is classified as Class III by its Spitzer colors. It has no counterpart in the ISO catalogue by Kaas et al. (2004). The source displays a variability of a factor of 2 in its X-ray counts and its X-ray spectrum (Table 2) is well fit by a $1-T$ model, with $N(\mathrm{H})=4.1 \times 10^{21} \mathrm{~cm}^{-2}, k T=1.3 \mathrm{keV}$, and $Z=0.11 Z_{\odot}$, typical of a Class III source.

\subsection{Source 11}

One of the brightest X-ray sources in the region, and bright in 2MASS, its Spitzer colors identify it as a Class III source; it has no counterpart in the ISO catalogue. A fit to its X-ray spectrum gives $k T=1.7 \mathrm{keV}$ and $N(\mathrm{H})=4.7 \times 10^{21} \mathrm{~cm}^{-2}$ (Table 2), also typical for a Class III source.

\subsection{Source 27}

A bright 2MASS source, classified on the basis of the Spitzer colors as Class III; it has no counterpart in the ISO catalogue. Its fitted absorbing column density, $N(\mathrm{H})=7.2 \times 10^{21} \mathrm{~cm}^{-2}$, and plasma temperature, $k T=1.1 \mathrm{keV}$ are typical for a Class III source.

\subsection{Source 34}

A bright 2MASS source already identified as a member of the Serpens cloud by Eiroa \& Casali (1992) ([EC92] 67), both the ISO and Spitzer colors indicate a Class II source. The X-ray light curve shows variability within a factor of $\simeq 2$, while the $\mathrm{X}$-ray spectrum is relatively hot, with $k T=3.2 \mathrm{keV}$, and $N(\mathrm{H})=7.6 \times$ $10^{21} \mathrm{~cm}^{-2}$.

\subsection{Source 38}

An object with Class III Spitzer colors, which has no counterpart in the ISO catalogue. The X-ray light curve shows a shortlasting, impulsive flare. The spectrum has a best fit temperature of $k T=2.5 \mathrm{keV}$ and a low absorption, $N(\mathrm{H})=0.5 \times 10^{21} \mathrm{~cm}^{-2}$. The low $N(\mathrm{H})$ value makes it likely that the source is at the surface of the Serpens cloud.

\subsection{Source 47}

The only optically bright star in our sample, HD 170545 , has photospheric colors compatible with an A0 spectral type (Straizys et al. 1996), and photometrically determined extinction $A_{V}=0.53$, compatible with Serpens membership. The source was already identified in the IR study by Strom et al. (1976). Spitzer colors indicate a Class III sources, consistent with a thin disk. Straizys et al. (1996) indicate that a fainter companion at 7 arcsec is present. The X-ray spectrum has a moderate temperature $(k T=0.6 \mathrm{keV})$ and a low absorption $N(\mathrm{H}) \leq 3 \times 10^{20}$, compatible with the optical absorption.

\subsection{Source 53}

This source is classified as a flat-spectrum YSO in the ISO study and by its Spitzer colors. It has a radio counterpart (Bontemps 1996) and was classified as a YSO by Eiroa \& Casali (1989) and Kaas et al. (2004). The light curve shows significant variability: a factor of three on the $20 \mathrm{ks}$ time scale. Its best fit plasma temperature $(k T=3.8 \mathrm{keV})$ and absorbing column density $\left(N(\mathrm{H})=4.2 \times 10^{22} \mathrm{~cm}^{-2}\right)$ indicate an other relatively hot and deeply embedded object.

\subsection{Source 65}

Classified as Class II in ISO study and as Class III by its Spitzer colors, this star already identified by Bontemps (1996), has a low best fit absorbing column density indicating that is probably in the surface of the cloud. The star shows significant variability (a factor of 3 over $40 \mathrm{ks}$ ) and a relatively high plasma temperature $(k T=2.95 \mathrm{keV})$.

\subsection{Source 67}

This source has no counterpart in the ISO catalogue but it is seen in the Spitzer observation and classified as Class III. It was first observed in the IR by Churchwell \& Koornneef (1986). A spectral fit with an absorbed 1T plasma gives $N(\mathrm{H})=0.8 \times$ $10^{22} \mathrm{~cm}^{-2}$ and $k T=2.6 \mathrm{keV}$.

\subsection{Source 69}

Also this source has no counterpart in the ISO catalogue, but it is classified as a Class II from its Spitzer colors. Its best-fit absorbing column density $\left(N(\mathrm{H})=1.2 \times 10^{22} \mathrm{~cm}^{-2}\right)$ is typical for the Serpens cloud. The best-fit plasma temperature is $k T=$ $1.0 \mathrm{keV}$.

\subsection{Source 73}

An object with Spitzer colors compatible with being Class II, with no counterpart in the ISO catalogue. The X-ray light curve shows a short-lasting, impulsive flare. The spectrum has a best fit temperature of $k T=1.0 \mathrm{keV}$ and and a typical absorption value of $N(\mathrm{H})=0.8 \times 10^{22} \mathrm{~cm}^{-2}$.

\subsection{Source 75}

A Class II object identified both in the ISO and Spitzer data. It has an absorption value below the average $(N(\mathrm{H})=0.5 \times$ $\left.10^{22} \mathrm{~cm}^{-2}\right)$ for this sample and a relatively high plasma temperature $(k T=2.8 \mathrm{keV})$. 
Table 5. X-ray sources detected with PwDETECT in the Chandra observation of the Serpens star forming region. For the sources with a $2 \mathrm{MASS}$ counterpart (within a search radius of 3 arcsec) the values of their magnitude in the $J, H$ and $K$ bands are also given, together with the radial distance from this counterpart $(r)$, the associations $(a)$ with Tycho 2 or USNOA-2.0 optical catalog sources, U or T respectively and their optical magnitude. The ISO field provides the source number and Class of the counterpart in the ISO catalogue by Kaas et al. (2004) (also within a search radius of 3 arcsec). The last three fields give the source identification numbers and classification in the Spitzer data: the first id is the source IR id and the second one the YSO id number. Class I/II and Class II/III indicate Flat Spectrum and Transition Disk objects, respectively. All the Spitzer counterparts are within 1 arcsec from the X-ray sources, unless their IR id is accompanied by $\dagger$ or is in between brackets. In the first case, although the matching distance is greater than $1^{\prime \prime}$, we are confident in the cross-identification reliability, in the second case some doubts remains (these are usually faint IR sources).

\begin{tabular}{|c|c|c|c|c|c|c|c|c|c|c|c|c|c|c|}
\hline$\overline{\mathrm{N}}$. & $\begin{array}{r}\text { RA(J200) } \\
\text { deg } \\
\end{array}$ & $\begin{array}{r}\text { Dec(J200) } \\
\text { deg } \\
\end{array}$ & $\begin{array}{r}\text { Count rate } \\
\mathrm{ks}^{-1} \\
\end{array}$ & $J$ & $H$ & $K$ & $\begin{array}{r}r \\
\operatorname{arcsec} \\
\end{array}$ & $\mathrm{a}$ & $B$ & $\overline{V V R}$ & $\overline{\overline{\mathrm{ISO}}}$ & $\overline{\overline{\mathrm{IR}}}$ & $\overline{\overline{Y S O}}$ & Class \\
\hline 1 & 182910.8 & 011202 & $1.07 \pm 0.20$ & 16.5 & 15.4 & 15.2 & 2.4 & 0 & - & - & - & - & - & - \\
\hline 2 & 182922.7 & 011033 & $3.18 \pm 0.23$ & 12.2 & 11.2 & 10.9 & 0.4 & $\mathrm{U}$ & 18.0 & 15.1 & - & 13836 & 221 & III \\
\hline 3 & 182927.7 & 011258 & $0.63 \pm 0.11$ & 9.7 & 9.5 & 9.4 & 1.0 & $\mathrm{~T}$ & 11.5 & 11.1 & - & $8974^{\dagger}$ & - & III \\
\hline 4 & 182928.2 & 011548 & $0.26 \pm 0.09$ & - & - & - & - & - & - & - & - & 57260 & - & - \\
\hline 5 & 182929.8 & 012119 & $0.48 \pm 0.14$ & - & - & - & - & - & - & - & - & 44904 & - & - \\
\hline 6 & 182929.8 & 012157 & $0.82 \pm 0.14$ & - & - & - & - & - & - & - & - & 57617 & 119 & II \\
\hline 7 & 182931.4 & 011956 & $1.23 \pm 0.15$ & - & - & - & - & - & - & - & - & 44113 & - & - \\
\hline 8 & 182931.8 & 011339 & $1.09 \pm 0.18$ & - & - & - & - & - & - & - & - & 40525 & - & \\
\hline 9 & 182932.0 & 011842 & $0.36 \pm 0.11$ & 11.6 & 9.8 & 8.6 & 0.3 & 0 & - & - & $159 \mathrm{I} / \mathrm{II}$ & 12134 & 36 & $\mathrm{I} / \mathrm{II}$ \\
\hline 10 & 182932.9 & 012240 & $0.69 \pm 0.18$ & - & - & - & - & - & - & - & - & 45772 & - & - \\
\hline 11 & 182933.1 & 011716 & $15.91 \pm 0.46$ & 11.4 & 10.3 & 10.0 & 0.2 & $\mathrm{U}$ & 17.9 & 15.0 & - & 14252 & 225 & III \\
\hline 12 & 182934.2 & 011703 & $0.08 \pm 0.05$ & - & - & - & - & - & - & - & - & - & - & - \\
\hline 13 & 182935.2 & 012339 & $4.15 \pm 0.27$ & - & - & - & - & - & - & - & - & 46388 & 50 & $\mathrm{I} / \mathrm{II}$ \\
\hline 14 & 182935.5 & 011654 & $0.23 \pm 0.08$ & - & - & - & - & - & - & - & - & - & - & - \\
\hline 15 & 182935.6 & 012311 & $0.29 \pm 0.11$ & - & - & - & - & - & - & - & - & - & - & - \\
\hline 16 & 182938.1 & 011023 & $0.25 \pm 0.09$ & - & - & - & - & - & - & - & - & - & - & - \\
\hline 17 & 182938.5 & 011450 & $0.11 \pm 0.05$ & - & - & - & - & - & - & - & - & (41344) & - & - \\
\hline 18 & 182939.9 & 011756 & $0.71 \pm 0.10$ & 14.5 & 12.8 & 11.9 & 0.1 & 0 & - & - & 202 II & 13040 & 94 & II \\
\hline 19 & 182941.1 & 011343 & $0.08 \pm 0.06$ & - & - & - & - & - & - & - & - & - & - & - \\
\hline 20 & 182942.1 & 011312 & $0.34 \pm 0.10$ & - & - & - & - & - & - & - & - & - & - & - \\
\hline 21 & 182942.3 & 011202 & $1.31 \pm 0.15$ & 16.8 & 14.4 & 12.9 & 0.1 & 0 & - & - & - & 13257 & 215 & III \\
\hline 22 & 182942.7 & 011401 & $0.11 \pm 0.05$ & - & - & - & - & - & - & - & - & 40962 & - & - \\
\hline 23 & 182944.6 & 011311 & $0.92 \pm 0.25$ & 13.3 & 12.2 & 11.8 & 0.3 & 0 & - & - & - & $13758^{\dagger}$ & 100 & II \\
\hline 24 & 182944.7 & 011003 & $0.18 \pm 0.08$ & 16.6 & 14.6 & 13.7 & 2.8 & 0 & - & - & - & (7965) & - & III \\
\hline 25 & 182945.1 & 012437 & $0.27 \pm 0.10$ & - & - & - & - & - & - & - & - & - & - & - \\
\hline 26 & 182946.3 & 010747 & $0.54 \pm 0.16$ & - & - & - & - & - & - & - & - & (37471) & - & - \\
\hline 27 & 182947.2 & 012234 & $3.14 \pm 0.22$ & 11.8 & 10.5 & 10.1 & 0.2 & $\mathrm{U}$ & 19.8 & 16.0 & - & 10876 & 199 & III \\
\hline 28 & 182949.6 & 011706 & $0.42 \pm 0.14$ & 16.9 & 15.7 & 12.7 & 0.2 & 0 & - & - & $254 \mathrm{I}$ & 10187 & 7 & I \\
\hline 29 & 182950.6 & 010858 & $0.79 \pm 0.12$ & 14.4 & 13.0 & 12.5 & 0.3 & 0 & - & - & - & $12228^{\dagger}$ & 209 & III \\
\hline 30 & 182951.2 & 011641 & $0.30 \pm 0.09$ & 16.7 & 15.1 & 13.0 & 1.2 & 0 & - & - & $265 \mathrm{I}$ & 42625 & 24 & I \\
\hline 31 & 182951.2 & 011320 & $0.11 \pm 0.05$ & 16.5 & 14.5 & 13.6 & 0.2 & 0 & - & - & - & 13144 & 95 & II \\
\hline 32 & 182952.2 & 011559 & $1.01 \pm 0.19$ & - & - & - & - & - & - & - & - & 42231 & 22 & I \\
\hline 33 & 182952.9 & 011456 & $0.12 \pm 0.06$ & - & - & - & - & - & - & - & 276 I & 41692 & 20 & I \\
\hline 34 & 182953.6 & 011702 & $6.11 \pm 0.28$ & 12.6 & 11.2 & 10.4 & 0.2 & $\mathrm{U}$ & 19.7 & 16.6 & 283 II & 10801 & 81 & II \\
\hline 35 & 182954.5 & 011447 & $0.29 \pm 0.09$ & 14.2 & 13.3 & 12.8 & 0.2 & 0 & - & - & - & 11909 & 87 & II \\
\hline 36 & 182955.4 & 011034 & $0.35 \pm 0.07$ & 14.0 & 13.4 & 12.9 & 0.1 & 0 & - & - & - & 11296 & 201 & III \\
\hline 37 & 182955.7 & 011431 & $0.50 \pm 0.08$ & 15.3 & 12.5 & 10.7 & 0.2 & 0 & - & - & 298 II & 12547 & 38 & $\mathrm{I} / \mathrm{II}$ \\
\hline 38 & 182956.2 & 011057 & $4.14 \pm 0.25$ & 12.5 & 11.7 & 11.4 & 0.2 & 0 & - & - & - & 11728 & 205 & III \\
\hline 39 & 182956.4 & 011218 & $0.11 \pm 0.04$ & 15.3 & 14.3 & 13.6 & 0.1 & 0 & - & - & - & (11699) & 204 & III \\
\hline 40 & 182956.6 & 011259 & $0.16 \pm 0.06$ & 15.0 & 12.8 & 11.5 & 0.3 & 0 & - & - & 304 II & 10636 & 80 & II \\
\hline 41 & 182956.7 & 011954 & $0.18 \pm 0.06$ & - & - & - & - & - & - & - & - & (57555) & - & - \\
\hline 42 & 182956.7 & 011239 & $0.40 \pm 0.08$ & 18.1 & 16.5 & 14.1 & 0.2 & 0 & - & - & 306 I & 8797 & 61 & II \\
\hline 43 & 182956.9 & 011446 & $0.28 \pm 0.08$ & 12.0 & 10.8 & 9.3 & 0.2 & 0 & - & - & $307 \mathrm{I} / \mathrm{II}$ & 13114 & 9 & I \\
\hline 44 & 182957.0 & 011248 & $2.93 \pm 0.20$ & 15.2 & 12.5 & 11.1 & 0.1 & 0 & - & - & 309 II & 11376 & 85 & II \\
\hline 45 & 182957.4 & 011450 & $1.28 \pm 0.12$ & 13.3 & 11.7 & 10.9 & 0.2 & 0 & - & - & - & 8506 & 190 & III \\
\hline 46 & 182957.6 & 011300 & $0.17 \pm 0.06$ & 16.5 & 14.1 & 13.1 & 0.2 & 0 & - & - & 312 I & 11670 & 8 & I \\
\hline 47 & 182957.7 & 011053 & $2.41 \pm 0.21$ & 8.1 & 8.1 & 8.2 & 0.1 & $\mathrm{~T}$ & 8.6 & 8.5 & - & 9456 & 192 & III \\
\hline 48 & 182957.7 & 011405 & $6.61 \pm 0.36$ & 12.2 & 9.2 & 7.2 & 0.4 & 0 & - & - & $314^{*} \mathrm{I} / \mathrm{II}$ & $10172^{\dagger}$ & 35 & $\mathrm{I} / \mathrm{II}$ \\
\hline $49^{\ddagger}$ & 182957.8 & 011407 & $0.27 \pm 0.13$ & - & - & - & 1.4 & 0 & - & - & - & - & - & - \\
\hline 50 & 182957.8 & 011532 & $1.64 \pm 0.26$ & 16.2 & 12.8 & 10.8 & 0.2 & 0 & - & - & 319 II & 10942 & 83 & II \\
\hline $51^{\text {常}}$ & 182957.8 & 011408 & $0.11 \pm 0.05$ & - & - & - & 2.4 & 0 & - & - & - & - & - & - \\
\hline
\end{tabular}

‡ sources associated with the elongated PSF of source 48 (SVS 20). * distance of ISO source greater than 3 arcsec. 
Table 5. continued.

\begin{tabular}{|c|c|c|c|c|c|c|c|c|c|c|c|c|c|c|}
\hline N. & $\begin{array}{r}\mathrm{RA}(\mathrm{J} 200) \\
\mathrm{deg}\end{array}$ & $\begin{array}{r}\operatorname{Dec}(\mathrm{J} 200) \\
\mathrm{deg}\end{array}$ & $\begin{array}{r}\text { Count rate } \\
\mathrm{ks}^{-1}\end{array}$ & $J$ & $H$ & $\bar{K}$ & $\begin{array}{r}r \\
\operatorname{arcsec}\end{array}$ & $\mathrm{a}$ & $\bar{B}$ & $V R$ & ISO & IR & YSO & Class \\
\hline 52 & 182957.8 & 011238 & $0.29 \pm 0.09$ & 17.2 & 14.4 & 11.7 & 0.2 & 0 & - & - & $318 \mathrm{I} / \mathrm{II}$ & 12355 & 37 & $\mathrm{I} / \mathrm{II}$ \\
\hline 53 & 182957.9 & 011251 & $6.7 \pm 0.50$ & 15.8 & 12.5 & 10.5 & 0.2 & 0 & - & - & $317 \mathrm{I} / \mathrm{II}$ & $8173^{\dagger}$ & 2 & I \\
\hline 54 & 182957.9 & 011246 & $44.99 \pm 0.76$ & 16.6 & 12.5 & 10.0 & 0.2 & 0 & - & - & - & 14647 & 105 & II \\
\hline 55 & 182958.2 & 011521 & $1.78 \pm 0.18$ & 13.1 & 11.2 & 9.9 & 0.2 & 0 & - & - & $321 \mathrm{II}$ & 7685 & 27 & $\mathrm{I} / \mathrm{II}$ \\
\hline 56 & 182958.5 & 011250 & $0.19 \pm 0.06$ & 18.1 & 14.8 & 12.3 & 0.3 & 0 & - & - & $322 \mathrm{I} / \mathrm{II}$ & $12962^{\dagger}$ & - & II \\
\hline 57 & 182959.1 & 011114 & $0.56 \pm 0.10$ & 16.9 & 16.2 & 14.4 & 0.2 & 0 & - & - & - & 10535 & 198 & II \\
\hline 58 & 182959.1 & 011120 & $0.31 \pm 0.11$ & 14.2 & 12.6 & 11.9 & 0.3 & 0 & - & - & - & 10161 & 196 & III \\
\hline 59 & 182959.2 & 011408 & $1.29 \pm 0.20$ & 11.8 & 10.4 & 9.5 & 0.2 & $\mathrm{U}$ & 19.7 & 16.9 & $328 \mathrm{II}$ & 8450 & 59 & II \\
\hline 60 & 182959.6 & 011158 & $0.71 \pm 0.11$ & 17.9 & 17.4 & 14.7 & 0.4 & 0 & - & - & $330 \mathrm{I}$ & 8176 & 3 & I \\
\hline 61 & 182959.6 & 011412 & $1.32 \pm 0.14$ & - & - & - & - & - & - & - & - & 41458 & 19 & I \\
\hline 62 & 183000.2 & 011403 & $0.15 \pm 0.05$ & 15.7 & 13.9 & 12.8 & 0.2 & 0 & - & - & - & 8029 & 28 & $\mathrm{I} / \mathrm{II}$ \\
\hline 63 & 183000.3 & 010944 & $0.53 \pm 0.14$ & - & - & - & - & - & - & - & - & 38922 & 110 & II \\
\hline 64 & 183000.5 & 012345 & $0.89 \pm 0.24$ & - & - & - & - & - & - & - & - & (57779) & - & - \\
\hline 65 & 183000.7 & 011340 & $6.04 \pm 0.28$ & 13.4 & 11.2 & 10.1 & 0.2 & 0 & - & - & 338 II & $13265^{\dagger}$ & 216 & III \\
\hline 66 & 183001.1 & 011324 & $0.83 \pm 0.11$ & 17.9 & 15.4 & 13.2 & 0.2 & 0 & - & - & $341 \mathrm{I} / \mathrm{II}$ & 8794 & 30 & I/II \\
\hline 67 & 183001.2 & 011503 & $5.58 \pm 0.34$ & 15.4 & 13.1 & 12.0 & 0.2 & 0 & - & - & - & 10085 & 195 & III \\
\hline 68 & 183001.4 & 011808 & $0.16 \pm 0.06$ & - & - & - & - & - & - & - & - & - & - & - \\
\hline 69 & 183003.4 & 011619 & $2.32 \pm 0.21$ & 12.3 & 11.1 & 10.4 & 0.1 & $\mathrm{U}$ & 18.9 & 15.0 & - & 10468 & 78 & II \\
\hline 70 & 183004.6 & 012233 & $2.21 \pm 0.23$ & - & - & - & - & - & - & - & - & 57713 & - & - \\
\hline 71 & 183004.9 & 011439 & $0.15 \pm 0.06$ & 13.7 & 12.7 & 12.2 & 0.4 & 0 & - & - & - & 13700 & 98 & II \\
\hline 72 & 183005.5 & 011425 & $0.20 \pm 0.07$ & 15.2 & 14.4 & 14.1 & 0.5 & 0 & - & - & - & 11452 & 203 & III \\
\hline 73 & 183006.1 & 010617 & $2.35 \pm 0.22$ & 12.9 & 11.8 & 11.2 & 0.5 & $\mathrm{U}$ & 19.0 & 16.5 & - & 10946 & 84 & II \\
\hline 74 & 183006.7 & 011216 & $1.02 \pm 0.13$ & - & - & - & - & - & - & - & - & (40517) & - & - \\
\hline 75 & 183007.7 & 011204 & $2.95 \pm 0.28$ & 12.3 & 10.8 & 10.1 & 0.1 & 0 & - & - & 366 II & 9882 & 73 & II \\
\hline 76 & 183008.2 & 011055 & $0.41 \pm 0.12$ & - & - & - & - & - & - & - & - & (39762) & - & - \\
\hline 77 & 183009.9 & 011707 & $0.16 \pm 0.07$ & - & - & - & - & - & - & - & - & - & - & - \\
\hline 78 & 183010.4 & 011006 & $0.31 \pm 0.10$ & - & - & - & - & - & - & - & - & 39349 & - & - \\
\hline 79 & 183011.1 & 011238 & $22.70 \pm 0.57$ & 13.3 & 12.5 & 12.0 & 0.1 & 0 & - & - & 393 II & 13765 & 101 & II \\
\hline 80 & 183011.1 & 011752 & $0.24 \pm 0.08$ & - & - & - & - & - & - & - & - & (43769) & - & - \\
\hline 81 & 183012.6 & 011227 & $0.84 \pm 0.12$ & - & - & - & - & - & - & - & - & 57165 & - & - \\
\hline 82 & 183016.2 & 011754 & $0.22 \pm 0.07$ & - & - & - & - & - & - & - & - & - & - & - \\
\hline 83 & 183018.2 & 011417 & $0.68 \pm 0.12$ & 13.3 & 11.8 & 10.9 & 0.7 & 0 & - & - & - & 14137 & 40 & $\mathrm{I} / \mathrm{II}$ \\
\hline 84 & 183022.4 & 012044 & $1.46 \pm 0.19$ & 13.1 & 12.2 & 11.9 & 0.5 & $\mathrm{U}$ & 18.8 & 16.4 & - & 12371 & 157 & II/III \\
\hline 85 & 183023.1 & 012009 & $0.85 \pm 0.14$ & 13.4 & 12.6 & 12.2 & 1.0 & $\mathrm{U}$ & 19.50 & 16.80 & - & 8080 & 128 & II/III \\
\hline
\end{tabular}


G. Giardino et al.: The onset of X-ray emission in young stellar objects, Online Material p 5

Table 6. The cross-identification of the sources below was obtained from the Simbad database, with a search radius of 5 arcsec. Identifier are as follow: [B96] Bontemps (1996), [CK86] Churchwell \& Koornneef (1986), [EC92] Eiroa \& Casali (1992), [GCN98] Giovannetti et al. (1998), [HB96] Hurt \& Barsony (1996), [KOB2004] Kaas et al. (2004), [KCM2004] Klotz et al. (2004), [P2003] Preibisch (2003), [SVS76] Strom, Vrba \& Strom (1976).

\begin{tabular}{|c|c|c|c|}
\hline$\overline{2}$ & 182922.7 & +011033 & [P2003] J182922.8+011032 \\
\hline 3 & 182927.7 & +011258 & $\mathrm{BD}+013686$ \\
\hline 9 & 182932.0 & +011842 & IRAS 18269+0116 - ESO-HA 279 \\
\hline 11 & 182933.1 & +011716 & [P2003] J182933.1+011716 \\
\hline 13 & 182935.2 & +012339 & [P2003] J182935.1+012338 \\
\hline 18 & 182939.9 & +011756 & [P2003] J182939.7+011754 \\
\hline 21 & 182942.3 & +011202 & [KCM2004] J182942.36+011201.9 \\
\hline 23 & 182944.6 & +011311 & [EC92] 11 \\
\hline 27 & 182947.2 & +012234 & [P2003] J182947.3+012234 \\
\hline 28 & 182949.6 & +011706 & [EC92] 38 \\
\hline 30 & 182951.2 & +011641 & [EC92] 53 \\
\hline 31 & 182951.2 & +011320 & [EC92] $51-$ [GCN98] 37 \\
\hline 33 & 182952.9 & +011456 & [GCN98] 53 \\
\hline 34 & 182953.6 & +011702 & [EC92] 67 \\
\hline 35 & 182954.5 & +011447 & [EC92] 70 \\
\hline 36 & 182955.4 & +011034 & [P2003] J182955.3+011034 \\
\hline 37 & 182955.7 & +011431 & [CK86] $9-$ [EC92] 74 \\
\hline 38 & 182956.2 & +011057 & [P2003] J182956.3+011056 \\
\hline 39 & 182956.4 & +011218 & [EC92] 77 - [SVS76] 1 \\
\hline 40 & 182956.6 & +011259 & [EC92] 79 \\
\hline 42 & 182956.7 & +011239 & [EC92] 80 \\
\hline 43 & 182956.9 & +011446 & [SVS76] $2-[$ CK86] $3-$ [EC92] 82 \\
\hline 44 & 182957.0 & +011248 & [EC92] $84-$ [B96] Serpens 5 \\
\hline 45 & 182957.4 & +011450 & [EC92] 86 \\
\hline 46 & 182957.6 & +011300 & [B96] Serpens $6-$ [EC92] 88 \\
\hline 47 & 182957.7 & +011053 & [SVS76] 19 \\
\hline 48 & 182957.7 & +011405 & [SVS76] $20-$ [GCN98] $98-[$ [GCN98] $99-$ [B96] Serpens $7-$ [EC92] $90-$ [KOB2004] 314 \\
\hline 50 & 182957.8 & +011532 & [CK86] 13 - EC93 \\
\hline 52 & 182957.8 & +011238 & [EC92] 94 \\
\hline 53 & 182957.9 & +011251 & [EC92] $95-$ [B96] Serpens 8 \\
\hline 54 & 182957.9 & +011246 & [B96] Serpens 8 \\
\hline 55 & 182958.2 & +011521 & [CK86] $4-$ [EC92] 97 \\
\hline 56 & 182958.5 & +011250 & [EC92] 98 \\
\hline 57 & 182959.1 & +011114 & HH $456: \mathrm{HH}$ \\
\hline 59 & 182959.2 & +011408 & [CK86] 8 - [EC92] 105 \\
\hline 60 & 182959.6 & +011158 & [HB96] PS 1 \\
\hline 61 & 182959.6 & +011412 & [GCN98] $122-$ [B96] Serpens 10 \\
\hline 62 & 183000.2 & +011403 & [EC92] 114 \\
\hline 63 & 183000.3 & +010944 & [KOB2004] 332 \\
\hline 65 & 183000.7 & +011340 & [EC92] $117-[$ [K86] $6-$ [B96] Serpens 13 \\
\hline 66 & 183001.1 & +011324 & [EC92] 121 \\
\hline 67 & 183001.2 & +011503 & [CK86] $12-$ [GCN98] 147 \\
\hline 69 & 183003.4 & +011619 & [EC92] $135-$ [EC92] 138 \\
\hline 70 & 183004.6 & +012233 & [P2003] J183004.7+012232 \\
\hline 71 & 183004.9 & +011439 & [EC92] 149 \\
\hline 72 & 183005.5 & +011425 & [EC92] 156 \\
\hline 73 & 183006.1 & +010617 & [P2003] J183006.4+010616 \\
\hline 74 & 183006.7 & +011216 & [P2003] J183006.7+011216 \\
\hline 75 & 183007.7 & +011204 & [KOB2004] 366 \\
\hline 79 & 183011.1 & +011238 & [KOB2004] 393 \\
\hline 83 & 183018.2 & +011417 & [P2003] J183018.3+011416 \\
\hline 84 & 183022.4 & +012044 & [P2003] J183022.4+012044 \\
\hline
\end{tabular}


G. Giardino et al.: The onset of X-ray emission in young stellar objects, Online Material p 6
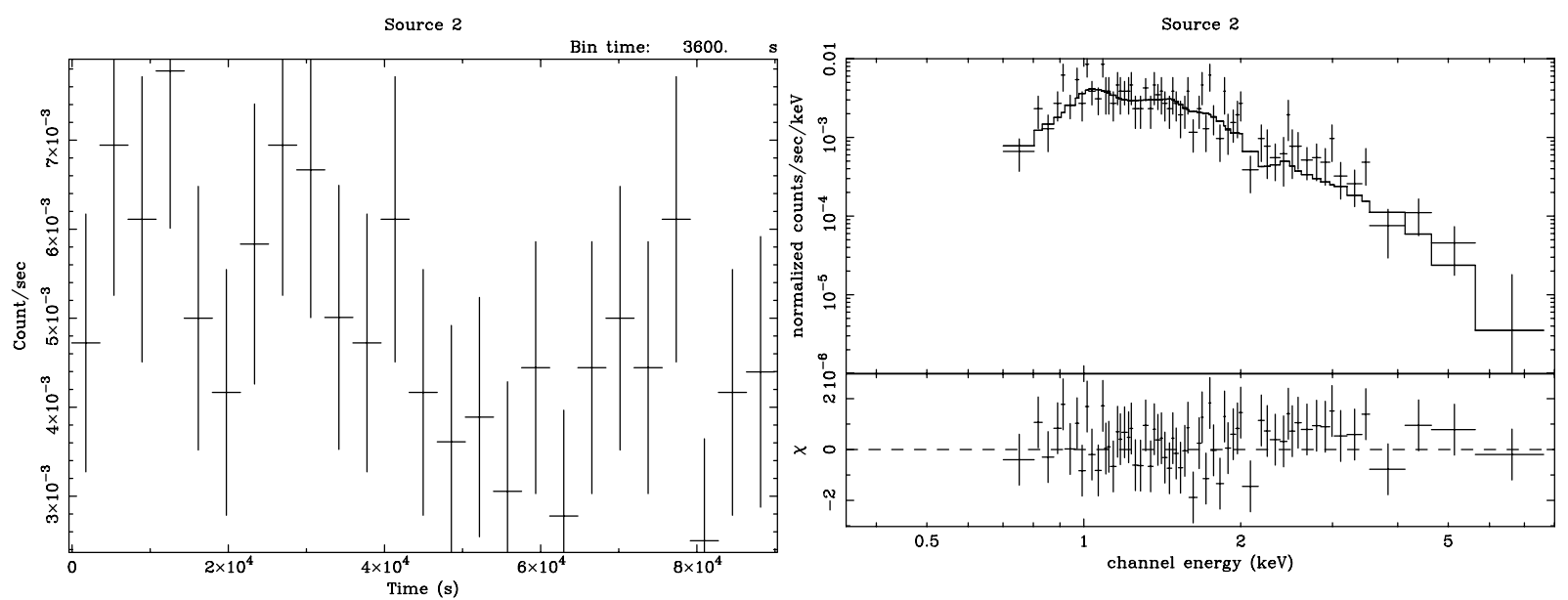

Start Time 13175 22:29:17:309 Stop Time 13176 22:29:17:309

Source 11

Source 11
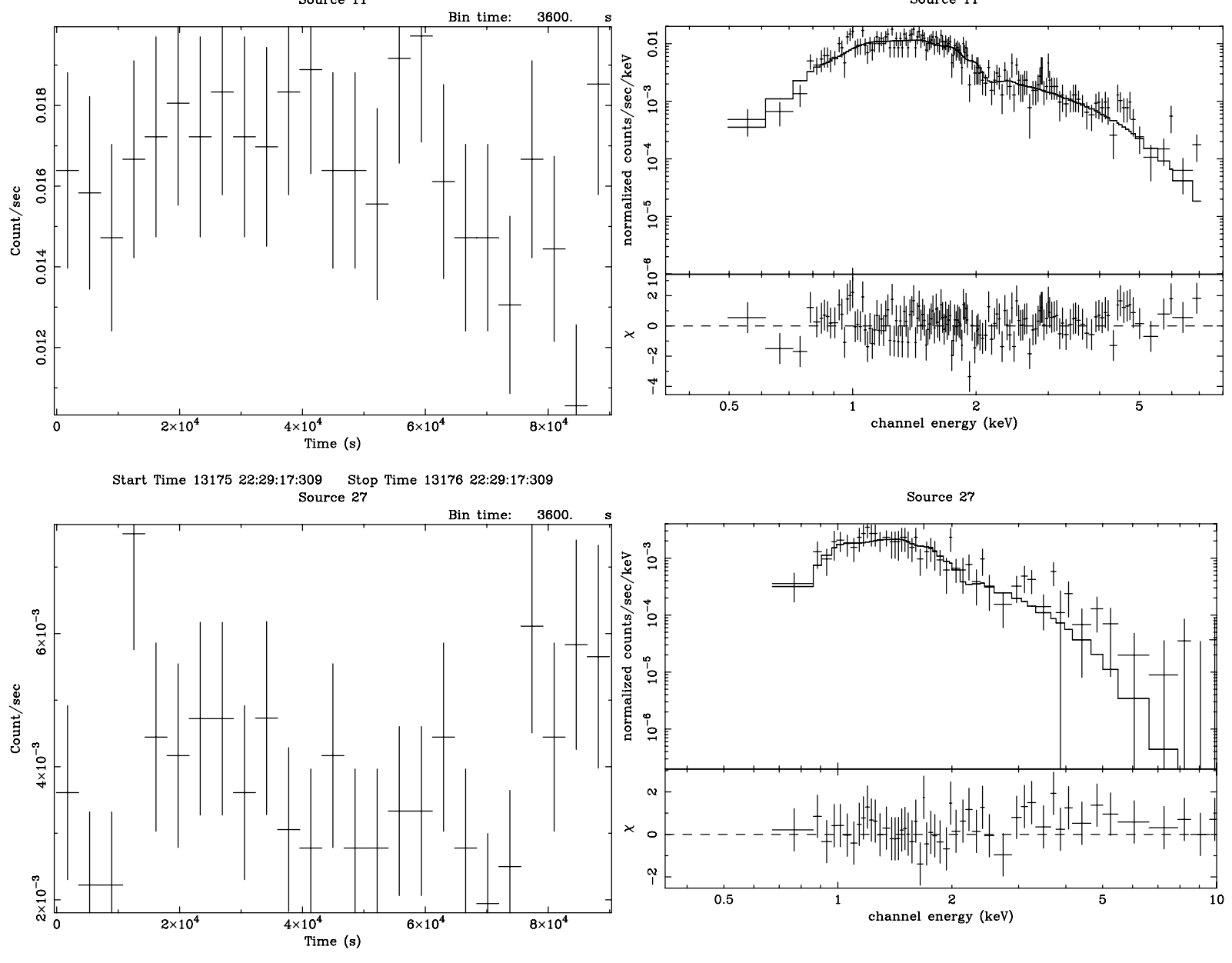

Source 27

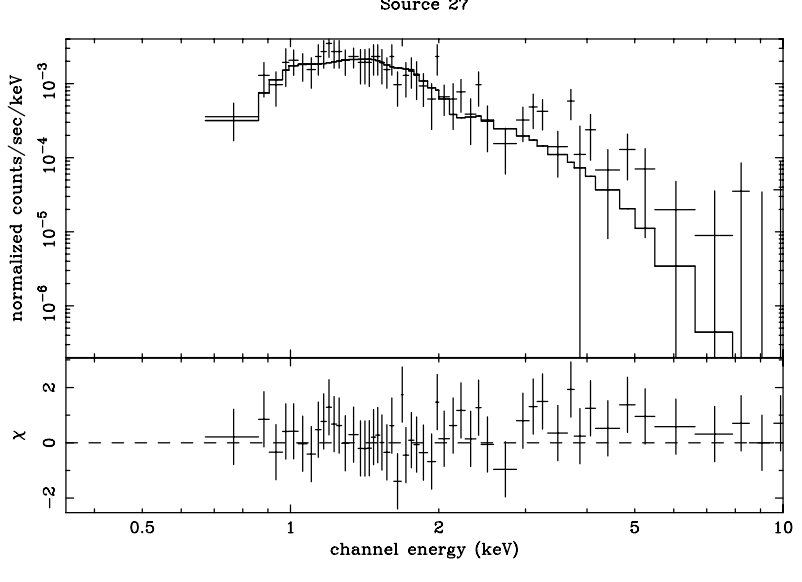

Fig. 13. Light curves and spectra with spectral fits of sources with more than $2 \mathrm{cts} \mathrm{ks}^{-1}$. 
G. Giardino et al.: The onset of X-ray emission in young stellar objects, Online Material $p 7$
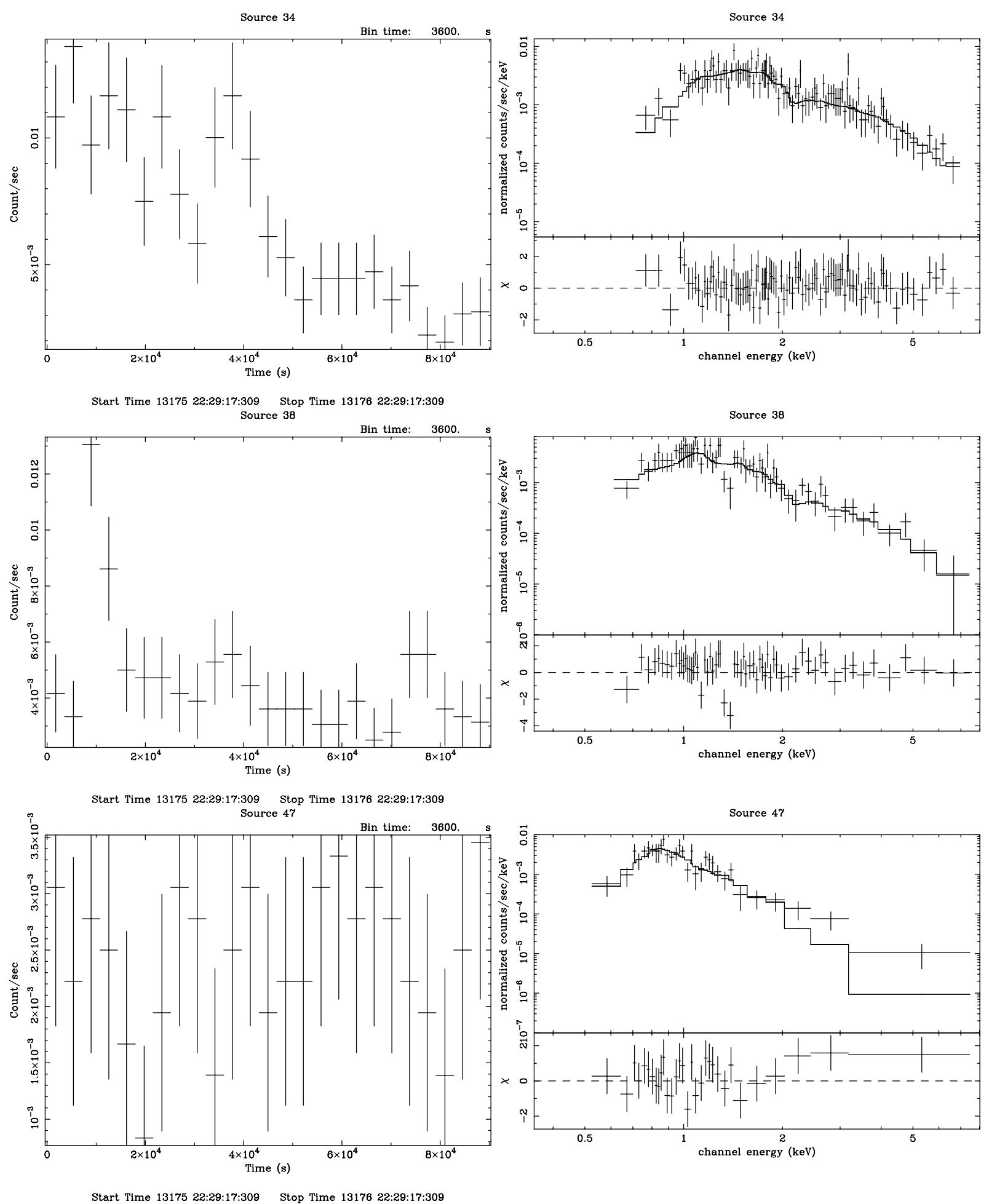

Fig. 13. continued. 
G. Giardino et al.: The onset of X-ray emission in young stellar objects, Online Material $p 8$
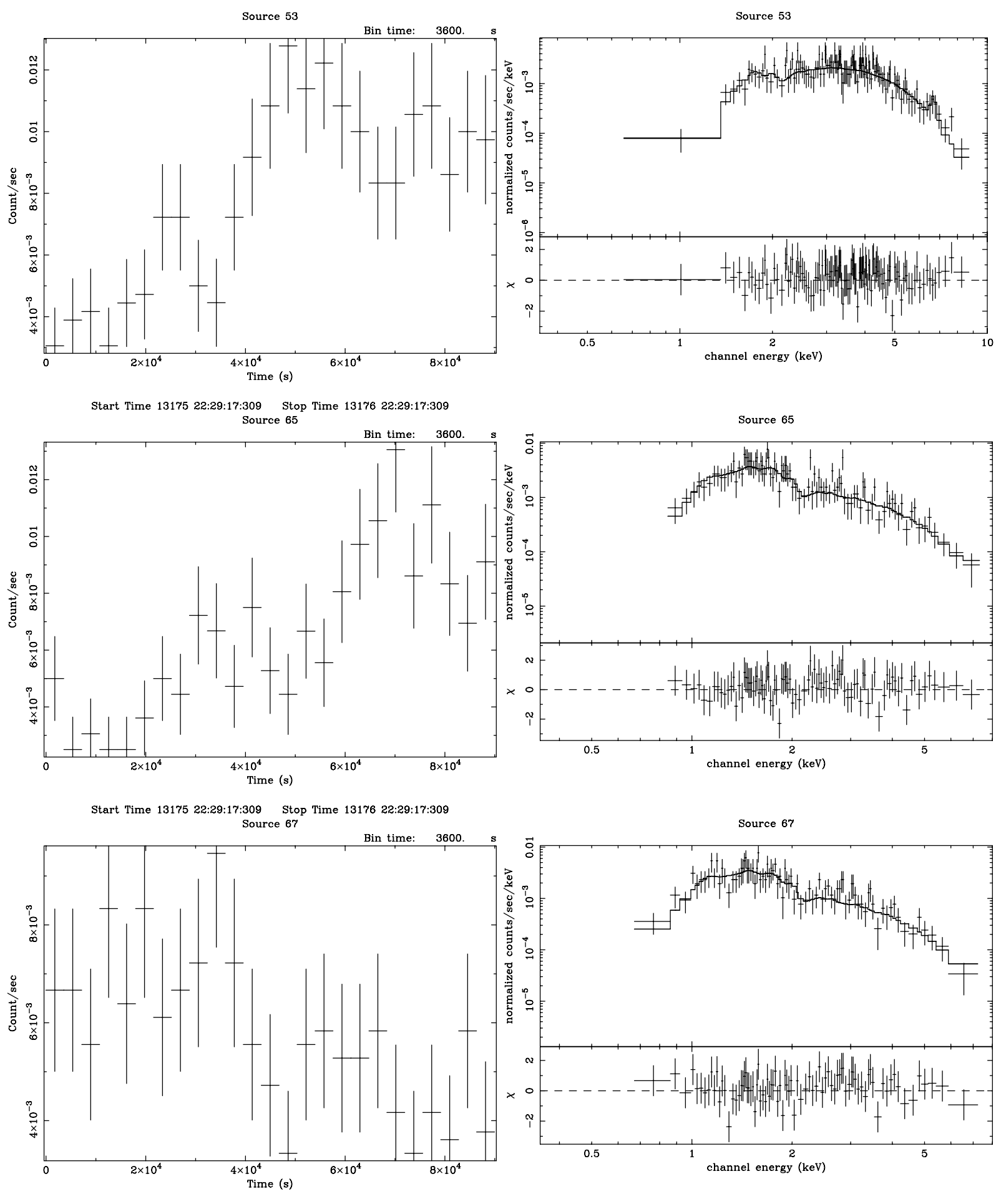

Fig. 13. continued. 
G. Giardino et al.: The onset of X-ray emission in young stellar objects, Online Material $p 9$
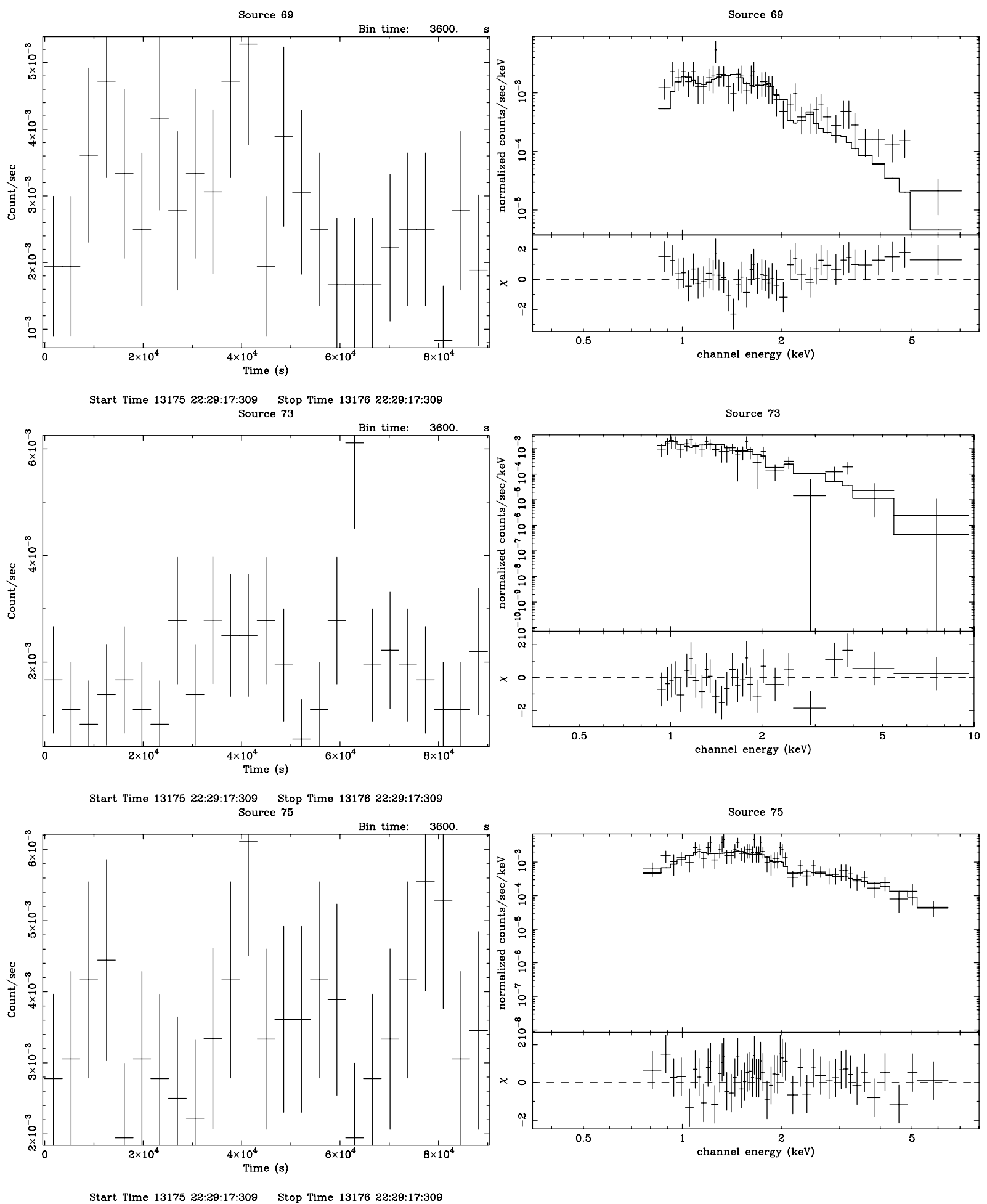

Fig. 13. continued. 